\title{
Effect of inhomogeneities on the expansion rate of the Universe
}

\author{
Edward W. Kolb* \\ Particle Astrophysics Center, Fermi National Accelerator Laboratory, Batavia, Illinois 60510-0500, USA \\ and Department of Astronomy and Astrophysics, Enrico Fermi Institute, \\ University of Chicago, Chicago, Illinois 60637-1433 USA \\ Sabino Matarrese $\oplus^{\dagger}$ \\ Dipartimento di Fisica "G. Galilei," Università di Padova, \\ and INFN, Sezione di Padova, via Marzolo 8, Padova I-35131, Italy \\ Alessio Notar: \\ Scuola Normale Superiore, Piazza dei Cavalieri 7, Pisa I-56126 and INFN, Sezione di Pisa, Italy \\ Antonio Riottd $\$$ \\ INFN, Sezione di Padova, via Marzolo 8, I-35131, Italy
}

(Dated: November 17, 2018)

\begin{abstract}
While the expansion rate of a homogeneous isotropic Universe is simply proportional to the squareroot of the energy density, the expansion rate of an inhomogeneous Universe also depends on the nature of the density inhomogeneities. In this paper we calculate to second order in perturbation variables the expansion rate of an inhomogeneous Universe and demonstrate corrections to the evolution of the expansion rate. While we find that the mean correction is small, the variance of the correction on the scale of the Hubble radius is sensitive to the physical significance of the unknown spectrum of density perturbations beyond the Hubble radius.

PACS numbers: $98.80 . \mathrm{Cq}$
\end{abstract}

\section{INTRODUCTION}

There is no more fundamental physical quantity in cosmology than the expansion rate of the Universe. In recent years the present value of the expansion rate, Hubble's constant, has been measured with increasing accuracy [1]. With the exploration of the Universe at redshifts of order unity, we now have information about the time evolution of the expansion rate 2]. A most surprising result is that the time evolution of the expansion rate does not seem to be described by a matter-dominated Friedmann-Lemaitre-Robertson-Walker (FLRW) cosmological model. The usual explanation for the discrepancy is that there is a new component of the energy density of the Universe, known as dark energy, that determines the recent evolution of the expansion rate. Of course all indications for dark energy are indirect; they all involve some form of the time evolution of the expansion rate.

Since the expansion rate of the Universe is of such fundamental importance, we must understand any possible effects that would result in an expansion rate different from the FLRW prediction. In this paper we study the change in the expansion rate due to perturbations of a homogeneous, isotropic, FLRW model. In particular, we perform a second-order calculation of the effect of inhomogeneities on the expansion rate. We only consider modifications to the expansion rate of a matter-dominated universe, although our results can be extended to a universe containing a mixture of matter and a cosmological constant. We find that the mean corrections are a few parts in $10^{5}$.

The expansion rate of a perturbed FLRW cosmology has been discussed in many works (although we believe ours is the first complete second-order calculation). Hui and Seljak [3] estimated the order of magnitude of the effect by considering a representative second-order term. They arrived at the correct mean order of magnitude of the result. Some recent works [4, 5] suggested that small-scale contributions could give a large correction, producing an apparent accelerated expansion of the universe. In particular Räsänen [5] suggested that due to ultraviolet sensitivity, one of the second-order terms could give large corrections depending on boundary conditions. As we will discuss in the conclusions, we find that if one employs the correct averaging procedure the result (at least for the terms we could

\footnotetext{
*Electronic address: rocky@fnal.gov

${ }^{\dagger}$ Electronic address: sabino.matarrese@pd.infn.it

${ }^{\ddagger}$ Electronic address: alessio.notari@sns.it

§Electronic address: antonio.riotto@pd.infn.it
} 
compute exactly using the second-order formalism) is well behaved in the ultraviolet and the term identified by Räsänen does not result in a large correction.

In addition to calculating the mean value of the expansion rate, we calculate the variance about the mean value. In calculating the variance we uncover an interesting infrared effect. At second order the expansion rate has a term proportional to $\varphi \nabla^{2} \varphi$, where $\varphi(\mathbf{x})$ is the peculiar gravitational potential, related to the density perturbations through the cosmological Poisson equation. If inflation is the origin of perturbations, then $\varphi$ should be a Gaussian random variable with zero mean. However, if the perturbation spectrum on super-Hubble-radius scales is no bluer than a Harrison-Zel'dovich spectrum, then the variance of $\varphi$ formally has an infrared singularity. ${ }^{1}$ Now if the value of $\varphi$ in our local Hubble volume is sufficiently large to modify the expansion rate, our perturbative expansion fails. Nevertheless, our results suggest that if the super-Hubble modes of $\varphi$ have physical significance, then a nonperturbative extension of our calculation could yield a most important modification to the Friedmann equation. One might even speculate that a complete treatment of the effect could explain the observed time dependence of the expansion rate on its own and obviate the need for the dark-energy assumption. Since that conjecture is beyond the perturbative calculation of this paper, we will postpone discussion of this point to a subsequent communication [6].

In the next section we discuss the general perturbative expansion. In Section III we calculate the corrections to the expansion rate in terms of metric fluctuations, and then express the results in terms of the density perturbation spectrum. In Section IV we present the numerical results. We then conclude, followed by two technical appendices.

\section{THE GENERAL FORMALISM}

In this section we describe how to treat the average properties of a perturbed Universe up to second order in the metric variables, including the effect of the inhomogeneous gravitational field on the homogeneous background field. We then discuss the proper definition of the average expansion rate and express the effect of inhomogeneities to second order as averages of density perturbations.

By "average," we mean the average over a spatial hypersurface at a given time. Clearly an average defined in this way depends on the chosen coordinate system, i.e., the gauge.

We will consider a Universe filled only by irrotational dust and choose the coordinates of an observer at rest with the dust (i.e., comoving coordinates), and with the same time coordinate for every point of the spatial hypersurface (i.e., synchronous coordinates). This system of coordinates can be chosen if the Universe is filled by a single pressureless component. Since the pressure vanishes, the only force acting on the particles is gravity, and the comoving world lines coincide with geodesics. (See Ref. 7] for a full discussion of this point.)

We will call $\tau$ the (conformal) time in this gauge, and $x^{i}$ the spatial coordinates, so that the metric has the form

$$
d s^{2}=a^{2}(\tau)\left[-d \tau^{2}+\gamma_{i j}\left(\tau, x^{i}\right) d x^{i} d x^{j}\right]
$$

We will perform the averages on constant- $\tau$ spatial hypersurfaces.

\section{A. First order}

The goal of this paper is a second-order calculation, but before embarking, let us recall the familiar linearized first-order result. The energy-momentum tensor and metric are expanded to first-order as

$$
\begin{aligned}
& T_{\mu \nu}=T_{\mu \nu}^{(0)}+T_{\mu \nu}^{(1)} \\
& g_{\mu \nu}=g_{\mu \nu}^{(0)}+g_{\mu \nu}^{(1)},
\end{aligned}
$$

where the superscript $(r)$ denotes the $r$-th order perturbation. By definition $T_{\mu \nu}^{(0)}$ and $g_{\mu \nu}^{(0)}$ are homogeneous and isotropic.

In the synchronous gauge, to first order the metric may be written in terms of a set of perturbation variables consisting of two scalars $\left(\psi^{(1)}, \chi^{(1)}\right)$, a vector $\left(\chi_{i}^{(1)}\right)$, and a tensor $\left(\chi_{i j}^{(1)}\right)$. The vector $\chi_{i}^{(1)}$ is transverse $\left(\partial^{i} \chi_{i}^{(1)}=0\right)$ and the tensor $\chi_{i j}^{(1)}$ is symmetric, transverse, and traceless $\left(\chi_{i j}^{(1)}=\chi_{j i}^{(1)}, \partial^{i} \chi_{i j}^{(1)}=0, \chi_{i}^{(1) i}=0\right)$. In terms of these variables the metric is

$$
\gamma_{i j}=\left(1-2 \psi^{(1)}\right) \delta_{i j}+D_{i j} \chi^{(1)}+\partial_{i} \chi_{j}^{(1)}+\partial_{j} \chi_{i}^{(1)}+\chi_{i j}^{(1)}
$$

\footnotetext{
${ }^{1}$ Presumably this infrared singularity is cut off because inflation did not last for an infinite period of time.
} 
where $D_{i j}=\partial_{i} \partial_{j}-\frac{1}{3} \nabla^{2} \delta_{i j}$.

The metric perturbations $\psi^{(1)}$ and $\chi^{(1)}$ may be expressed in terms of the peculiar gravitational potential $\varphi(\mathbf{x})$, which is related to $\delta^{(1)}$, the first-order density perturbation, by the Poisson equation,

$$
\nabla^{2} \varphi=\frac{\kappa^{2}}{2} a^{2} \rho^{(0)} \delta^{(1)}
$$

Ignoring metric perturbations that decay in time, for a matter-dominated Universe $\psi^{(1)}$ and $\chi^{(1)}$ are given by (see Ref. $[8])^{2}$

$$
\begin{aligned}
\psi^{(1)}(\mathbf{x}, \tau) & =\frac{5}{3} \varphi(\mathbf{x})+\frac{\tau^{2}}{18} \nabla^{2} \varphi(\mathbf{x}) \\
\chi^{(1)}(\mathbf{x}, \tau) & =-\frac{1}{3} \tau^{2} \varphi(\mathbf{x}) .
\end{aligned}
$$

To first order we will neglect vector modes, as they do not arise in conventional perturbation-generation mechanisms such as inflation. We will also assume the tensor mode amplitude is small.

In calculating spatial averages we will also require $\sqrt{\gamma}$, where $\gamma$ is the determinant of the spatial metric. To first order [8],

$$
\sqrt{\gamma}=1+\frac{1}{2} \gamma^{(1)}(\mathbf{x}, \tau)=1-3 \psi^{(1)}(\mathbf{x}, \tau)=1-5 \varphi(\mathbf{x})-\frac{\tau^{2}}{6} \nabla^{2} \varphi(\mathbf{x}) .
$$

Whether calculating to first order or second order, when calculating the spatial average $\langle\cdots\rangle$ of a quantity $\mathcal{O}\left(\tau, x^{i}\right)$ one must fix a system of coordinates in which to express $\mathcal{O}\left(\tau, x^{i}\right)$, and then integrate with the proper integration measure over $d^{3} x$ at fixed $\tau$. For all orders we adopt the definition ${ }^{3}$

$$
\langle\mathcal{O}\rangle(\tau) \equiv \frac{\int d^{3} x \sqrt{\gamma\left(\tau, x^{i}\right)} \mathcal{O}\left(\tau, x^{i}\right)}{\int d^{3} x \sqrt{\gamma\left(\tau, x^{i}\right)}},
$$

where the domain of integration is some large volume. If $\mathcal{O}^{(0)}$ is a homogeneous quantity (i.e., it does not depend on $x^{i}$, but only on $\tau$ ), then simply $\left\langle\mathcal{O}^{(0)}\right\rangle=\mathcal{O}^{(0)}$. In the first-order calculation, if $\mathcal{O}^{(1)}$ is already a first order quantity we may set $\gamma=1$, and $\left\langle\mathcal{O}^{(1)}\right\rangle$ becomes

$$
\left\langle\mathcal{O}^{(1)}\right\rangle=\frac{\int d^{3} x \mathcal{O}^{(1)}\left(\tau, x^{i}\right)}{\int d^{3} x}
$$

To first order, the Einstein equations are

$$
G_{\mu \nu}^{(0)}+G_{\mu \nu}^{(1)}=\kappa^{2}\left(T_{\mu \nu}^{(0)}+T_{\mu \nu}^{(1)}\right)
$$

which yield upon averaging the 00 component

$$
G_{00}^{(0)}=\kappa^{2}\left\langle T_{00}\right\rangle-\left\langle G_{00}^{(1)}\right\rangle .
$$

Here we see that $\kappa^{-2}\left\langle G_{00}^{(1)}\right\rangle$ may be interpreted as an extra component to the stress-energy tensor. ${ }^{4}$

For the unperturbed FLRW cosmology, the expansion rate $a^{\prime} / a^{2}$ is found from the $0-0$ component of the Einstein equations. For the first-order perturbed FLRW model

$$
3\left(\frac{a^{\prime}}{a^{2}}\right)^{2}=\kappa^{2}\langle\rho\rangle-a^{-2}\left\langle G_{00}^{(1)}\right\rangle
$$

\footnotetext{
2 The perturbation variable $\phi$ in Ref. 8 corresponds to our variable $\psi$, and vice versa.

3 Note that this definition is appropriate for scalar quantities. It will also be used to average $G_{00}$ which is a scalar under the residual gauge freedom.

4 This effect is sometimes incorrectly described as a "backreaction." Technically, it is not a backreaction; while the inhomogeneities modify the expansion rate of the Universe, they are not produced by the expansion of the universe.
} 
where $\rho=\rho^{(0)}+\rho^{(1)}=a^{-2} T_{00}$. This is the basic point. In a perturbed FLRW cosmology, $\dot{a} / a=a^{\prime} / a^{2}$ is $n o t$ $\sqrt{\kappa^{2}\left\langle\rho^{(0)}\right\rangle / 3} .^{5}$ However, one must be careful to find the true variables that describe the evolution of the averaged background. It is not clear that the quantity $a^{\prime} / a^{2}$ describes the physical Hubble flow. The correct quantity to describe the Hubble flow may be found from the evolution of $\langle\rho\rangle$.

We know that for a homogeneous isotropic Universe there are two independent equations that govern the dynamics of the expansion. So we have to find the two independent equations that describe the evolution of averaged physical quantities such as like $\langle\rho\rangle$. For the unperturbed model we may augment the $0-0$ equation with the continuity equation, $D_{\mu} T^{\mu 0}=0$. This gives for a perfect pressureless fluid (dust)

$$
\frac{1}{a} \rho^{\prime}=\dot{\rho}=-\theta \rho
$$

where $\theta \equiv D_{\mu} u^{\mu}$ and $u^{\mu}$ is the fluid four velocity. Eq. (12) is true to all orders. For an unperturbed FLRW Universe with scale factor $a$, this immediately gives $\rho^{\prime} / \rho=-3 a^{\prime} / a$, which results in $\rho \propto a^{-3}$.

For the perturbed model, we want to expand Eq. (12) to first order and then average it. This will give us the effective scale factor for the dilution of the matter density. In addition to the expansion of $\rho$, we expand $\theta$ as

$$
\theta=\theta^{(0)}+\theta^{(1)}
$$

and obtain

$$
\frac{1}{a}\left\langle\rho^{(0) \prime}+\rho^{(1) \prime}\right\rangle=-\left\langle\left(\theta^{(0)}+\theta^{(1)}\right)\left(\rho^{(0)}+\rho^{(1)}\right)\right\rangle
$$

Using the fact that $\left\langle\rho^{(1) \prime}\right\rangle=\left\langle\rho^{(1)}\right\rangle^{\prime}$ we are left with

$$
\frac{1}{a} \frac{\langle\rho\rangle^{\prime}}{\langle\rho\rangle}=-\langle\theta\rangle
$$

This tells us that in a first-order perturbed Universe the average matter density is diluted with expansion according to $\langle\rho\rangle^{-1} d\langle\rho\rangle / d t=-\langle\theta\rangle$, which in general is not equivalent to $\langle\rho\rangle^{-1} d\langle\rho\rangle / d t=-3 \dot{a} / a$.

An alternative way to express the result is to define a new scale factor, $a_{V}$, such that $\langle\rho\rangle \propto a_{V}^{-3}$. It is clear that $a_{V}$ must be defined by

$$
\frac{\dot{a}_{V}}{a_{V}} \equiv \frac{1}{3}\langle\theta\rangle
$$

And so the true scale factor is $a_{V}$ and not $a$, at least for the dilution of matter (see, e.g., [9]).

The physical quantity of interest is $\langle\theta\rangle$. It is given by

$$
\langle\theta\rangle=\theta^{(0)}+\left\langle\theta^{(1)}\right\rangle=3 \frac{a^{\prime}}{a^{2}}+\left\langle\theta^{(1)}\right\rangle .
$$

We will define a $\delta \theta$, and express $\theta$ as

$$
\langle\theta\rangle=3 \sqrt{\frac{\kappa^{2}\langle\rho\rangle}{3}}+\langle\delta \theta\rangle=3 H\left(1+\frac{\langle\delta \theta\rangle}{3 H}\right) .
$$

Two important notational points: We have defined $H \equiv \sqrt{\kappa^{2}\langle\rho\rangle / 3}$ (not as $\left.a^{\prime} / a^{2}\right)$; and we have defined $\langle\delta \theta\rangle$ as the difference between $\langle\theta\rangle$ and $3 H$ (not as the difference between $\langle\theta\rangle$ and $\theta^{(0)}$ ).

To calculate $\langle\delta \theta\rangle / 3 H$, we can express Eq. (11) in the form

$$
\frac{a^{\prime}}{a^{2}}=\left(\frac{\kappa^{2}\langle\rho\rangle}{3}-\frac{\left\langle G_{00}^{(1)}\right\rangle}{3 a^{2}}\right)^{1 / 2} \simeq H\left(1-\frac{\left\langle G_{00}^{(1)}\right\rangle}{6 a^{2} H^{2}}\right)
$$

\footnotetext{
${ }^{5}$ Here the overdot stands for $d / d t$, where $t$ is related to $\tau$ by $a d \tau=d t$.
} 
where of course the second equality holds if the corrections are small. Combining Eqs. (18) and (19), we find

$$
\frac{\langle\delta \theta\rangle}{3 H}=\frac{\left\langle\theta^{(1)}\right\rangle}{3 H}-\frac{\left\langle G_{00}^{(1)}\right\rangle}{6 a^{2} H^{2}} .
$$

It will turn out that in the first-order calculation $g_{\mu \nu}^{(1)}$ will appear only as a spatial gradient in the final expression for $\langle\delta \theta\rangle$. This means that the physical result is insensitive to the choice of the normalization of $\left\langle g_{\mu \nu}^{(1)}\right\rangle$, since we could always add a constant to $g_{\mu \nu}^{(1)}$ to make $\left\langle g_{\mu \nu}^{(1)}\right\rangle$ anything we please. The cancellation of the non-gradient first-order terms in Eq. (20) seems accidental, but in Appendix $\mathrm{A}$ we derive an expression for $\langle\delta \theta\rangle$ that at first order only contains spatial derivatives of $g_{\mu \nu}^{(1)}$, which do not change if we shift $g_{\mu \nu}^{(1)}$ by a constant (or even a time-dependent renormalization).

\section{B. Second order}

The spatial metric $\gamma_{i j}$ is expanded up to second order (neglecting first order vector and tensor perturbations) $\operatorname{as}^{6}$

$$
\gamma_{i j}=\left(1-2 \psi^{(1)}-\psi^{(2)}\right) \delta_{i j}+D_{i j}\left(\chi^{(1)}+\frac{1}{2} \chi^{(2)}\right)+\frac{1}{2}\left(\partial_{i} \chi_{j}^{(2)}+\partial_{j} \chi_{i}^{(2)}+\chi_{i j}^{(2)}\right) .
$$

The functions $\psi^{(r)}, \chi^{(r)}, \chi_{i}^{(r)}$, and $\chi_{i j}^{(r)}$ represent the $r$ th-order perturbation of the metric. Vector and tensor modes have been included here at second order as they are dynamically generated by the non-linear evolution of purely scalar perturbations [8].

It will turn out that $\psi^{(2)}(\mathbf{x}, \tau)$ is the only second-order term for which we will require the explicit form. For a matter-dominated Universe the metric perturbation $\psi^{(2)}(\mathbf{x}, \tau)$ is obtained similarly to Ref. [8]

$$
\psi^{(2)}(\mathbf{x}, \tau)=-\frac{50}{9} \varphi^{2}-\frac{5 \tau^{2}}{54} \varphi^{, k} \varphi_{, k}+\frac{\tau^{4}}{252}\left(\left(\nabla^{2} \varphi\right)^{2}-\frac{10}{3} \varphi^{, k i} \varphi_{, k i}\right) .
$$

The first term arises from a primordial epoch of inflation and can be computed as follows. There exists a second-order extension of the well-known gauge-invariant variable $\zeta$, the curvature perturbation on uniform density hypersurfaces. In terms of $\mathcal{H}=a^{\prime} / a$, to first order it is given by $\zeta^{(1)}=\psi^{(1)}+\mathcal{H} \delta^{(1)} \rho / \rho^{\prime}$. To second order, $\zeta=\zeta^{(1)}+(1 / 2) \zeta^{(2)}$, where $\zeta^{(2)}$ remains constant on superhorizon scales in the case in which only adiabatic perturbations are present. In standard single-field inflation, $\zeta^{(2)}$ is generated during inflation and its value is given by $\zeta^{(2)} \simeq-2\left(\zeta^{(1)}\right)^{2}[10$, 11]. Since in the synchronous gauge and on superhorizon scales $\zeta^{(1)} \simeq \psi^{(1)}=5 \varphi / 3$ and $\zeta^{(2)} \simeq \psi^{(2)}$, one readily concludes that the primordial contribution to the second-order metric perturbation $\psi^{(2)}$ is given by the constant term $-50 \varphi^{2} / 9$ and it also propagates to the coefficients of the term proportional to $\tau^{2}$.

In the second-order calculation care must be taken in defining the spatial average. Again, if $\mathcal{O}$ is a homogeneous quantity, then simply $\left\langle\mathcal{O}^{(0)}\right\rangle=\mathcal{O}^{(0)}$. If $\mathcal{O}$ is already a second-order quantity, then we can take $\gamma=1$, both in the numerator and in the denominator. But in the second-order calculation of $\left\langle\mathcal{O}^{(1)}\right\rangle$ we must remember to include $\sqrt{\gamma}$. Using Eq. (6),

$$
\begin{aligned}
\left\langle\mathcal{O}^{(1)}\right\rangle & =\frac{\int d^{3} x \sqrt{\gamma\left(\tau, x^{i}\right)} \mathcal{O}^{(1)}\left(\tau, x^{i}\right)}{\int d^{3} x \sqrt{\gamma\left(\tau, x^{i}\right)}}=\frac{\int d^{3} x\left(1-3 \psi^{(1)}\right) \mathcal{O}^{(1)}\left(\tau, x^{i}\right)}{\int d^{3} x\left(1-3 \psi^{(1)}\right)} \\
& =\frac{\int d^{3} x \mathcal{O}^{(1)}-3 \int d^{3} x \psi^{(1)} \mathcal{O}^{(1)}}{\int d^{3} x-3 \int d^{3} x \psi^{(1)}}=\left\langle\mathcal{O}^{(1)}\right\rangle_{1}-3\left\langle\psi^{(1)} \mathcal{O}^{(1)}\right\rangle+3\left\langle\psi^{(1)}\right\rangle_{1}\left\langle\mathcal{O}^{(1)}\right\rangle_{1},
\end{aligned}
$$

where we have introduced the notation $\langle\cdots\rangle_{1}$ for a first-order term to denote the spatial average with the factor $\sqrt{\gamma}=1$, i.e., $\left\langle\mathcal{O}^{(1)}\right\rangle_{1}(\tau) \equiv \int d^{3} x \mathcal{O}^{(1)}\left(\tau, x^{i}\right) / \int d^{3} x$.

So the correct second-order averaging procedure gives

$$
\begin{aligned}
\left\langle\mathcal{O}^{(0)}\right\rangle & =\mathcal{O}^{(0)} \\
\left\langle\mathcal{O}^{(1)}\right\rangle & =\left\langle\mathcal{O}^{(1)}\right\rangle_{1}-3\left\langle\psi^{(1)} \mathcal{O}^{(1)}\right\rangle+3\left\langle\psi^{(1)}\right\rangle_{1}\left\langle\mathcal{O}^{(1)}\right\rangle_{1} \\
\left\langle\mathcal{O}^{(2)}\right\rangle & =\frac{\int d^{3} x \mathcal{O}^{(2)}\left(\tau, x^{i}\right)}{\int d^{3} x} .
\end{aligned}
$$

\footnotetext{
${ }^{6}$ From now on spatial indices will be raised and lowered by the background metric $\delta_{i j}$.
} 
As an illustration of the subtleties in averaging, if at first order $\left\langle g_{\mu \nu}^{(1)}\right\rangle_{1}=0$, where the first-order averaging prescription is defined in Eq. (8), at second order the averaging prescription for a first-order quantity is defined by Eq. (24b), and $\left\langle g_{\mu \nu}^{(1)}\right\rangle$ need not vanish.

Now we perform a second-order expansion. We first expand the energy-momentum tensor and the metric as

$$
\begin{aligned}
T_{\mu \nu} & =T_{\mu \nu}^{(0)}+T_{\mu \nu}^{(1)}+T_{\mu \nu}^{(2)} \\
g_{\mu \nu} & =g_{\mu \nu}^{(0)}+g_{\mu \nu}^{(1)}+g_{\mu \nu}^{(2)},
\end{aligned}
$$

where again the superscript $(r)$ represents the $r$ th-order perturbation. Again we will take $T_{\mu \nu}^{(0)}$ and $g_{\mu \nu}^{(0)}$ to be homogeneous and isotropic, so $T_{\mu \nu}^{(0)}=\left\langle T_{\mu \nu}^{(0)}\right\rangle$ and $g_{\mu \nu}^{(0)}=\left\langle g_{\mu \nu}^{(0)}\right\rangle$. Note that this is not equivalent to the statement that $T_{\mu \nu}^{(0)}=\left\langle T_{\mu \nu}\right\rangle$ and $g_{\mu \nu}^{(0)}=\left\langle g_{\mu \nu}\right\rangle$.

Expanding the Einstein tensor $G_{\mu \nu}$ to second order yields several types of terms. The first type are the zeroth-order terms, denoted $G_{\mu \nu}^{(0)}$, which only involve $g_{\mu \nu}^{(0)} . G_{\mu \nu}^{(0)}$ will be homogeneous since $g_{\mu \nu}^{(0)}$ is homogeneous by definition. Then there are first-order terms, $G_{\mu \nu}^{(1)}$, which involve a single power of $g_{\mu \nu}^{(1)}$, possibly combined with $g_{\mu \nu}^{(0)}$. There are two types of second-order terms. The first type, denoted by $G_{\mu \nu}^{(11)}$, involves squares of $g_{\mu \nu}^{(1)}$. The second type of second-order terms, denoted $G_{\mu \nu}^{(2)}$, involves $g_{\mu \nu}^{(2)}$.

Now we consider the Einstein equations

$$
G_{\mu \nu}^{(0)}+G_{\mu \nu}^{(1)}+G_{\mu \nu}^{(11)}+G_{\mu \nu}^{(2)}=\kappa^{2}\left(T_{\mu \nu}^{(0)}+T_{\mu \nu}^{(1)}+T_{\mu \nu}^{(2)}\right) .
$$

We proceed by averaging Eq. (26). This yields

$$
G_{00}^{(0)}=\kappa^{2}\left\langle T_{00}\right\rangle-\left\langle G_{00}^{(1)}+G_{00}^{(11)}+G_{00}^{(2)}\right\rangle .
$$

From Eq. (27) we see that now $\kappa^{-2}\left\langle G_{00}^{(1)}+G_{00}^{(11)}+G_{00}^{(2)}\right\rangle$ may be interpreted as an extra component to the stressenergy tensor. To second order, the $0-0$ component of the perturbed FLRW model gives

$$
3\left(\frac{a^{\prime}}{a^{2}}\right)^{2}=\kappa^{2}\langle\rho\rangle-a^{-2}\left\langle G_{00}^{(1)}+G_{00}^{(11)}+G_{00}^{(2)}\right\rangle .
$$

Again we see that $\dot{a} / a=a^{\prime} / a^{2}$ is $n o t \kappa^{2} \rho^{(0)} / 3$.

Now let us consider the second-order expression for the evolution of $\langle\rho\rangle$. We again use the continuity equation, Eq. (12), and expand $\rho$ and $\theta$ as

$$
\begin{aligned}
& \rho=\rho^{(0)}+\rho^{(1)}+\rho^{(2)} \\
& \theta=\theta^{(0)}+\theta^{(1)}+\theta^{(11)}+\theta^{(2)} .
\end{aligned}
$$

The result is a bit more complicated than the first-order result:

$$
\frac{1}{a}\left\langle\rho^{(0) \prime}+\rho^{(1) \prime}+\rho^{(2) \prime}\right\rangle=-\left\langle\left(\theta^{(0)}+\theta^{(1)}+\theta^{(11)}+\theta^{(2)}\right)\left(\rho^{(0)}+\rho^{(1)}+\rho^{(2)}\right)\right\rangle .
$$

Using the fact that $\left\langle\rho^{(1) \prime}\right\rangle=\left\langle\rho^{(1)}\right\rangle^{\prime}-3\left\langle\rho^{(1)}\right\rangle_{1}\left\langle\psi^{(1)^{\prime}}\right\rangle_{1}+3\left\langle\psi^{(1) \prime} \rho^{(1)}\right\rangle$, the left-hand side of Eq. (30) becomes

$$
\frac{1}{a}\left\langle\rho^{(0) \prime}+\rho^{(1) \prime}+\rho^{(2) \prime}\right\rangle=\frac{1}{a}\langle\rho\rangle^{\prime}-\frac{3}{a}\left\langle\psi^{(1) \prime}\right\rangle_{1}\left\langle\rho^{(1)}\right\rangle_{1}+\frac{3}{a}\left\langle\psi^{(1) \prime} \rho^{(1)}\right\rangle .
$$

We may also express the right-hand side of Eq. (30) as

$$
-\left\langle\left(\theta^{(0)}+\theta^{(1)}+\theta^{(11)}+\theta^{(2)}\right)\left(\rho^{(0)}+\rho^{(1)}+\rho^{(2)}\right)\right\rangle=-\langle\theta\rangle\langle\rho\rangle+\left\langle\theta^{(1)}\right\rangle_{1}\left\langle\rho^{(1)}\right\rangle_{1}-\left\langle\rho^{(1)} \theta^{(1)}\right\rangle .
$$

Equating Eqs. (31) and (32) we obtain

$$
\frac{1}{a}\langle\rho\rangle^{\prime}-\frac{3}{a}\left\langle\psi^{(1) \prime}\right\rangle_{1}\left\langle\rho^{(1)}\right\rangle_{1}+\frac{3}{a}\left\langle\psi^{(1) \prime} \rho^{(1)}\right\rangle=-\langle\theta\rangle\langle\rho\rangle+\left\langle\theta^{(1)}\right\rangle_{1}\left\langle\rho^{(1)}\right\rangle_{1}-\left\langle\rho^{(1)} \theta^{(1)}\right\rangle .
$$


Since to first order a simple calculation yields $\theta^{(1)}=-3 \psi^{(1)^{\prime}} / a$, we are left with

$$
\frac{1}{a} \frac{\langle\rho\rangle^{\prime}}{\langle\rho\rangle}=-\langle\theta\rangle
$$

This tells us that as in a first-order perturbed universe, in a second-order perturbed Universe the average matter density is diluted with expansion according to $\langle\rho\rangle^{-1} d\langle\rho\rangle / d t=-\langle\theta\rangle$, which in general is not equivalent to $\langle\rho\rangle^{-1} d\langle\rho\rangle / d t=$ $-3 \dot{a} / a$. Note that it was crucial to define the averages with the $\sqrt{\gamma}$ factor; otherwise we would not have discovered the right quantity to describe the scaling of $\langle\rho\rangle$.

Again, the physical quantity of interest is $\langle\theta\rangle$, given by

$$
\langle\theta\rangle=\theta^{(0)}+\left\langle\theta^{(1)}\right\rangle+\left\langle\theta^{(11)}\right\rangle+\left\langle\theta^{(2)}\right\rangle=3 \frac{a^{\prime}}{a^{2}}+\left\langle\theta^{(1)}\right\rangle+\left\langle\theta^{(11)}\right\rangle+\left\langle\theta^{(2)}\right\rangle .
$$

We emphasize again that $H \equiv \sqrt{\kappa^{2}\langle\rho\rangle / 3}$ (and not $a^{\prime} / a^{2}$ ).

The goal of this paper is to calculate $\langle\delta \theta\rangle / 3 H$. To do so, we can express Eq. (28) in the form

$$
\frac{a^{\prime}}{a^{2}}=\left(\frac{\kappa^{2}\langle\rho\rangle}{3}-\frac{\left\langle G_{00}^{(1)}+G_{00}^{(11)}+G_{00}^{(2)}\right\rangle}{3 a^{2}}\right)^{1 / 2} \simeq H\left(1-\frac{\left\langle G_{00}^{(1)}+G_{00}^{(11)}+G_{00}^{(2)}\right\rangle}{6 a^{2} H^{2}}-\frac{\left\langle G_{00}^{(1)}\right\rangle^{2}}{72 a^{4} H^{4}}\right)
$$

where of course the second equality holds if the corrections are small. Using Eq. (36), we find

$$
\frac{\langle\delta \theta\rangle}{3 H}=\frac{\left\langle\theta^{(1)}+\theta^{(11)}+\theta^{(2)}\right\rangle}{3 H}-\frac{\left\langle G_{00}^{(1)}+G_{00}^{(11)}+G_{00}^{(2)}\right\rangle}{6 a^{2} H^{2}}-\frac{\left\langle G_{00}^{(1)}\right\rangle^{2}}{72 a^{4} H^{4}} .
$$

It will turn out that $g_{\mu \nu}^{(2)}$ will appear only as a spatial gradient in the final expression for $\langle\delta \theta\rangle$. This means that now the physical result is insensitive to the choice of the normalization of $\left\langle g_{\mu \nu}^{(2)}\right\rangle$, since we could always add a term that is spatially constant. The cancellation of the non-gradient second-order terms in Eq. (37) seems accidental, but again, in Appendix $\mathrm{A}$ we derive an expression for $\langle\delta \theta\rangle$ that explicitly only contains spatial derivatives of $g_{\mu \nu}^{(2)}$, which do not change if we shift $g_{\mu \nu}^{(2)}$ by a term that is spatially constant (or even a time-dependent normalization).

\section{COMPUTATION OF $\langle\delta \theta\rangle / 3 H$ IN THE SYNCHRONOUS GAUGE}

The relevant quantity which we want to calculate is $\langle\delta \theta\rangle / 3 H$. The simplest way to proceed is to perform the computation directly in the synchronous gauge. The synchronous coordinates are more physical for our purposes. If the calculation is performed in the Poisson gauge it is necessary to perform a complex calculation (as we do in Appendix (B) to express the result in synchronous coordinates (see also Refs. [5] and [12]).

Moreover, in general for second-order calculations, the synchronous gauge turns out to be very convenient, since here the scalar perturbations do not have nonlocal terms that appear in other gauges such as the Poisson gauge. Also, for matter $u^{\mu}$ in this gauge takes the trivial form $u^{\mu}=a^{-1}(\tau)(1, \overrightarrow{0})$, so the only terms that have to be computed are the Christoffel symbols in the covariant derivative

$$
\theta=D_{\mu} u^{\mu}=\partial_{\mu} u^{\mu}+\Gamma_{\mu \alpha}^{\alpha} u^{\mu}=3 \frac{a^{\prime}}{a^{2}}+\frac{\delta \Gamma_{00}^{0}+\delta \Gamma_{0 i}^{i}}{a} .
$$

Moreover, in the synchronous gauge, to second order $\delta \Gamma_{0}^{00}=0$ [11]. Finally, the computation of the perturbation of $\theta$ with respect to its background value consists of finding just the trace of $\Gamma_{0 j}^{i}$. From [1] ,

$$
\begin{aligned}
\frac{\left\langle\theta^{(1)}+\theta^{(11)}+\theta^{(2)}\right\rangle}{3 H} & =\frac{1}{3 H}\left\langle\delta \Gamma_{0 i}^{i}\right\rangle=\frac{1}{3 H a}\left\langle\delta^{(1)} \Gamma_{0 i}^{i}+\delta^{(2)} \Gamma_{0 i}^{i}\right\rangle \\
& =\frac{1}{a H}\left\langle-\psi^{(1) \prime}-\frac{1}{2} \psi^{(2) \prime}-2 \psi^{(1)} \psi^{(1) \prime}+\frac{1}{18} \nabla^{2} \chi^{(1)} \nabla^{2} \chi^{(1) \prime}-\frac{1}{6} \chi^{(1), k j} \chi_{, j k}^{(1) \prime}\right\rangle \\
& =\frac{1}{a^{2} H^{2}}\left\langle-\frac{2}{9} \nabla^{2} \varphi-\frac{20}{27} \varphi \nabla^{2} \varphi+\frac{5}{27} \varphi_{, i} \varphi^{, i}-\frac{\tau^{2}}{63}\left(\nabla^{2} \varphi\right)^{2}-\frac{4 \tau^{2}}{189} \varphi^{, i j} \varphi_{, i j}\right\rangle .
\end{aligned}
$$


In the last equation we expressed the result in terms of the initial perturbation $\varphi$, using the time evolution of the relevant perturbation variables in the synchronous gauge given in Eqs. (5) and (22).

The simplest way to find $G_{00}$ is to realize that $a^{-2}\left(G_{00}^{(1)}+G_{00}^{(11)}+G_{00}^{(2)}\right)=\kappa^{2}\left(\rho^{(1)}+\rho^{(2)}\right)$, and use the explicit solutions for $\rho^{(1)}$ and $\rho^{(2)}$ from Ref. [8], but using for $\psi^{(2)}$ the expression augmented with the $\varphi^{2}$ as in Eq. (22). In the matter-dominated universe,

$$
\begin{aligned}
-\frac{\left\langle G_{00}^{(1)}+G_{00}^{(11)}+G_{00}^{(2)}\right\rangle}{6 a^{2} H^{2}} & =\frac{1}{a^{2} H^{2}}\left\langle-\frac{1}{3} \nabla^{2} \varphi-\frac{10}{27} \varphi \nabla^{2} \varphi+\frac{55}{54} \varphi_{, i} \varphi^{, i}-\frac{5 \tau^{2}}{126}\left(\nabla^{2} \varphi\right)^{2}-\frac{\tau^{2}}{63} \varphi^{, i j} \varphi_{, i j}\right\rangle \\
-\frac{\left\langle G_{00}^{(1)}\right\rangle^{2}}{72 a^{4} H^{4}} & =-\frac{1}{a^{2} H^{2}} \frac{\tau^{2}}{72}\left\langle\nabla^{2} \varphi\right\rangle\left\langle\nabla^{2} \varphi\right\rangle .
\end{aligned}
$$

The value of $\delta \theta / 3 H$ is found by summing Eqs. (39) and (40).

We must now perform the appropriate spatial average. In $\delta \theta$ there is only one first-order term; the rest of the terms are second order. The first-order term must be averaged using the procedure of Eq. (23), while the other terms are averaged using $\sqrt{\gamma}=1$. The result is

$$
\begin{aligned}
\frac{\langle\delta \theta\rangle}{3 H}= & \frac{1}{a^{2} H^{2}}\left[-\frac{5}{9}\left\langle\nabla^{2} \varphi\right\rangle_{1}+\frac{5}{3}\left(\left\langle\varphi \nabla^{2} \varphi\right\rangle+\frac{13}{18}\left\langle\varphi, i \varphi^{, i}\right\rangle\right)+\frac{\tau^{2}}{27}\left(\left\langle\left(\nabla^{2} \varphi\right)^{2}\right\rangle-\left\langle\varphi^{, i j} \varphi_{, i j}\right\rangle\right)\right. \\
& \left.-\frac{25}{9}\langle\varphi\rangle_{1}\left\langle\nabla^{2} \varphi\right\rangle_{1}-\frac{23 \tau^{2}}{216}\left\langle\nabla^{2} \varphi\right\rangle_{1}\left\langle\nabla^{2} \varphi\right\rangle_{1}\right] .
\end{aligned}
$$

\section{A. Evaluation of $\langle\delta \theta\rangle$ in terms of the matter power spectrum}

We now proceed to express the averages in terms of the matter power spectrum. The procedure is to fix a spherical domain of radius $R$ with volume $V(R)$. From Eq. (7) and the definition of $\langle\cdots\rangle_{1}$, all of the averages in Eq. (41) involve integrals of the form

$$
\langle\cdots\rangle \equiv \frac{1}{V(R)} \int_{V(R)}(\cdots) d^{3} x
$$

For calculational convenience we will employ a Gaussian window function and assume $V(R)$ is a spherically symmetric volume with volume element $d V=4 \pi r^{2} \exp \left(-r^{2} / 2 R^{2}\right) d r$ and volume $V(R)=(2 \pi)^{3 / 2} R^{3}$. The Fourier transform of the window functions is

$$
W(k R)=V^{-1}(R) \int d^{3} x e^{-r^{2} / 2 R^{2}} \exp (i \vec{k} \cdot \vec{x})=e^{-k^{2} R^{2} / 2} .
$$

Of course as $k R \rightarrow 0, W(k R) \rightarrow 1$.

We wish to evaluate the typical expected value of $\theta$ averaged over this sphere. By "typical expected value" we mean the ensemble average. The metric fluctuation $\varphi$ is treated as a Gaussian variable with zero mean (of which we know the $N$-point correlation functions) that takes random values over different "realizations" of volumes $V(R)$. In other words, we calculate the typical value of a quantity for a region of radius $R$ as the statistical mean over many different similar regions. We will indicate this statistical average with a bar: $\overline{\langle\cdots\rangle}$.

We will express $\varphi$ and its derivatives in terms of a Fourier integral, so

$$
\varphi=\int \frac{d^{3} k}{(2 \pi)^{3}} \varphi_{\vec{k}} e^{i \vec{k} \cdot \vec{x}}, \quad \varphi_{, i}=\int \frac{d^{3} k}{(2 \pi)^{3}} i k_{i} \varphi_{\vec{k}} e^{i \vec{k} \cdot \vec{x}}, \quad \nabla^{2} \varphi=-\int \frac{d^{3} k}{(2 \pi)^{3}} k^{2} \varphi_{\vec{k}} e^{i \vec{k} \cdot \vec{x}}, \quad \text { etc. }
$$

The Fourier components $\varphi_{\vec{k}}$ satisfy

$$
\begin{aligned}
\overrightarrow{\varphi_{\vec{k}}}= & 0 \\
\frac{\varphi_{\vec{k}_{1}} \varphi_{\vec{k}_{2}}}{=} & (2 \pi)^{3} \delta^{(3)}\left(\overrightarrow{k_{1}}+\overrightarrow{k_{2}}\right) P_{\varphi}\left(k_{1}\right) \\
\frac{\varphi_{\vec{k}_{1}} \varphi_{\vec{k}_{2}} \varphi_{\vec{k}_{3}} \varphi_{\vec{k}_{4}}}{=} & (2 \pi)^{6}\left\{\delta^{(3)}\left(\vec{k}_{1}+\vec{k}_{2}\right) \delta^{(3)}\left(\vec{k}_{3}+\vec{k}_{4}\right) P_{\varphi}\left(k_{1}\right) P_{\varphi}\left(k_{3}\right)\right. \\
& \left.+\left[\delta^{(3)}\left(\vec{k}_{1}+\vec{k}_{3}\right) \delta^{(3)}\left(\vec{k}_{2}+\vec{k}_{4}\right)+\delta^{(3)}\left(\vec{k}_{1}+\vec{k}_{4}\right) \delta^{(3)}\left(\vec{k}_{2}+\vec{k}_{3}\right)\right] P_{\varphi}\left(k_{1}\right) P_{\varphi}\left(k_{2}\right)\right\}
\end{aligned}
$$


where $P_{\varphi}(k)=\left|\varphi_{\vec{k}}\right|^{2}$. From Eq. (4) we can express $P_{\varphi}(k)$ in terms of the matter power spectrum as

$$
P_{\varphi}(k) \equiv \frac{9 \pi^{2}}{2} a^{4} H^{4} \frac{\Delta^{2}(k, a)}{k^{7}},
$$

where $\Delta^{2}(k, a)$ is the (dimensionless) power spectrum of the matter density fluctuations.

Let us first consider $\overline{\langle\varphi\rangle_{1}}$ and $\overline{\left\langle\nabla^{2} \varphi\right\rangle_{1}}$. Clearly from Eq. (45a), $\overline{\langle\varphi\rangle_{1}}=0$ and $\overline{\left\langle\nabla^{2} \varphi\right\rangle_{1}}=0$. However this does not imply that $\langle\varphi\rangle_{1}=0$ or $\left\langle\nabla^{2} \varphi\right\rangle_{1}=0$ over any individual volume of radius $R$. The question is the magnitude of typical departures from the mean values, which corresponds to the statistical variance of our quantities. As we will show, it is intuitively clear that if the radius $R$ is big enough, this variance will go to zero. So the effect of variance could be important. We will return to the calculation of the variances of the different terms after completing the calculation of the mean values.

Next, consider $\overline{\left\langle\varphi \nabla^{2} \varphi\right\rangle}$. Passing to Fourier space, we have

$$
\begin{aligned}
& \overline{\left\langle\varphi \nabla^{2} \varphi\right\rangle}=-\int_{V(R)} \frac{d^{3} x}{V(R)} \frac{d^{3} k_{1}}{(2 \pi)^{3}} \frac{d^{3} k_{2}}{(2 \pi)^{3}}\left(\frac{k_{1}^{2}+k_{2}^{2}}{2}\right) \overline{\varphi_{\overrightarrow{k_{1}}} \varphi_{\overrightarrow{k_{2}}}} \exp \left[i\left(\vec{k}_{1}+\vec{k}_{2}\right) \cdot \vec{x}\right] \\
& =-\int \frac{d^{3} k_{1}}{(2 \pi)^{3}} \frac{d^{3} k_{2}}{(2 \pi)^{3}}\left(\frac{k_{1}^{2}+k_{2}^{2}}{2}\right) \frac{\varphi_{\vec{k}_{1}} \varphi_{\vec{k}_{2}}}{} W\left(\left|\overrightarrow{k_{1}}+\overrightarrow{k_{2}}\right| R\right) \text {. }
\end{aligned}
$$

Making use of Eq. 45b), we find

$$
\overline{\left\langle\varphi \nabla^{2} \varphi\right\rangle}=-\int \frac{d^{3} k}{(2 \pi)^{3}} k^{2} P_{\varphi}(k) .
$$

Using Eq. (46), we can express $\overline{\left\langle\varphi \nabla^{2} \varphi\right\rangle}$ in its final form. In the same manner we find the means for the other terms. The result is

$$
\begin{aligned}
\overline{\langle\varphi\rangle_{1}} & =0 \\
\overline{\left\langle\nabla^{2} \varphi\right\rangle_{1}} & =0 \\
\overline{\left\langle\varphi \nabla^{2} \varphi\right\rangle} & =-\frac{9}{4} a^{4} H^{4} \int_{0}^{\infty} \frac{d k}{k^{3}} \Delta^{2}(k, a) \\
\overline{\left\langle\varphi_{, i} \varphi^{, i}\right\rangle} & =+\frac{9}{4} a^{4} H^{4} \int_{0}^{\infty} \frac{d k}{k^{3}} \Delta^{2}(k, a) \\
\overline{\left\langle\left(\nabla^{2} \varphi\right)^{2}\right\rangle} & =+\frac{9}{4} a^{4} H^{4} \int_{0}^{\infty} \frac{d k}{k} \Delta^{2}(k, a) \\
\overline{\left\langle\varphi^{, i j} \varphi_{, i j}\right\rangle} & =+\frac{9}{4} a^{4} H^{4} \int_{0}^{\infty} \frac{d k}{k} \Delta^{2}(k, a) \\
\overline{\langle\varphi\rangle_{1}\left\langle\nabla^{2} \varphi\right\rangle_{1}} & =-\frac{9}{4} a^{4} H^{4} \int_{0}^{\infty} \frac{d k}{k^{3}} \Delta^{2}(k, a) W^{2}(k R) \\
\frac{\left\langle\nabla^{2} \varphi\right\rangle_{1}\left\langle\nabla^{2} \varphi\right\rangle_{1}}{4} & =+\frac{9}{4} a^{4} H^{4} \int_{0}^{\infty} \frac{d k}{k} \Delta^{2}(k, a) W^{2}(k R) .
\end{aligned}
$$

Of course in the limit $R \rightarrow \infty$, Eqs. (49g) and (49h) vanish. Therefore, the entire second-order calculation gives

$$
\frac{\overline{\langle\delta \theta\rangle}}{3 H}=-\frac{25}{24} a^{2} H^{2} \int_{0}^{\infty} \frac{d k}{k^{3}} \Delta^{2}(k, a)+\frac{25}{4} a^{2} H^{2} \int_{0}^{\infty} \frac{d k}{k^{3}} \Delta^{2}(k, a) W^{2}(k R)-\frac{23}{96} \int_{0}^{\infty} \frac{d k}{k} \Delta^{2}(k, a) W^{2}(k R),
$$

where we have used $\tau=2 / a H$ appropriate for a matter-dominated Universe. We will give numerical results in the next section.

Now for the variances of selected terms. The variance is defined as

$$
\operatorname{Var}[\langle\cdots\rangle]=\overline{(\langle\cdots\rangle-\overline{\langle\cdots\rangle})^{2}} \text {. }
$$

For instance,

$$
\begin{aligned}
\operatorname{Var}\left[\langle\varphi\rangle_{1}\right] & =\frac{9}{4} a^{4} H^{4} \int_{0}^{\infty} \frac{d k}{k^{5}} \Delta^{2}(k, a) W^{2}(k R) \\
\operatorname{Var}\left[\left\langle\nabla^{2} \varphi\right\rangle_{1}\right] & =\frac{9}{4} a^{4} H^{4} \int_{0}^{\infty} \frac{d k}{k} \Delta^{2}(k, a) W^{2}(k R) .
\end{aligned}
$$


The variances of other terms are more complicated, but straightforward to derive. For instance,

$$
\operatorname{Var}\left[\left\langle\varphi \nabla^{2} \varphi\right\rangle+\frac{13}{18}\left\langle\varphi_{, i} \varphi^{, i}\right\rangle\right]=\frac{1}{(2 \pi)^{6}} \int d^{3} k_{1} d^{3} k_{2} \frac{1}{2}\left[k_{1}^{2}+k_{2}^{2}+\frac{13}{9} \vec{k}_{1} \cdot \vec{k}_{2}\right]^{2} W^{2}\left(\left|\overrightarrow{k_{1}}+\overrightarrow{k_{2}}\right| R\right) P_{\varphi}\left(k_{1}\right) P_{\varphi}\left(k_{2}\right)
$$

The angular integrals can be expressed in terms of a filter function, defined in general as

$$
J^{(l)}\left(k_{1}, k_{2}, R\right) \equiv \int_{-1}^{1} d \mu \mu^{l} W^{2}\left(\sqrt{k_{1}^{2}+k_{2}^{2}+2 k_{1} k_{2} \mu} R\right) .
$$

For a Gaussian window function the filter expression can be expressed in terms of incomplete $\Gamma$ functions as

$$
\begin{aligned}
J^{(l)}\left(k_{1}, k_{2}, R\right) & =\int_{-1}^{1} d \mu \mu^{l} e^{-\left(k_{1}^{2}+k_{2}^{2}+2 \mu k_{1} k_{2}\right) R^{2}} \\
& =\frac{\exp \left[-\left(k_{1}^{2}+k_{2}^{2}\right) R^{2}\right]\left[\Gamma\left(l+1,-2 R^{2} k_{1} k_{2}\right)-\Gamma\left(l+1,2 R^{2} k_{1} k_{2}\right)\right]}{2^{l+1} R^{2(l+1)} k_{1}{ }^{l+1} k_{2}{ }^{l+1}} .
\end{aligned}
$$

We will make use of the fact that for $k_{1} R \rightarrow 0$ and $k_{2} R \rightarrow 0, J^{(0)}\left(k_{1}, k_{2}, R\right) \rightarrow 2$.

Using the Gaussian filter, Eq. (53) becomes

$$
\begin{aligned}
\operatorname{Var}\left[\left\langle\varphi \nabla^{2} \varphi\right\rangle+\frac{13}{18}\left\langle\varphi, i \varphi^{, i}\right\rangle\right]= & \left(\frac{9}{8} a^{4} H^{4}\right)^{2} \int_{0}^{\infty} \frac{d k_{1}}{k_{1}^{3}} \Delta^{2}\left(k_{1}, a\right) \int_{0}^{\infty} \frac{d k_{2}}{k_{2}^{3}} \Delta^{2}\left(k_{2}, a\right)\left[\left(\frac{k_{1}^{2}}{k_{2}^{2}}+\frac{k_{2}^{2}}{k_{1}^{2}}+2\right) J^{(0)}\left(k_{1}, k_{2}, R\right)\right. \\
& \left.+\frac{26}{9}\left(\frac{k_{1}}{k_{2}}+\frac{k_{2}}{k_{1}}\right) J^{(1)}\left(k_{1}, k_{2}, R\right)+\frac{169}{81} J^{(2)}\left(k_{1}, k_{2}, R\right)\right] .
\end{aligned}
$$

It is interesting that the integral is not well behaved in the infrared. As discussed in the next section, for a HarrisonZel'dovich spectrum $\Delta^{2}(k) \propto k^{4}$, so the term proportional to $J^{(0)}$ has an infrared divergence:

$$
\begin{aligned}
\operatorname{Var}\left[\left\langle\varphi \nabla^{2} \varphi\right\rangle\right]_{\infty} & =\left(\frac{9}{8} a^{4} H^{4}\right)^{2} \int_{0}^{\infty} \frac{d k_{1}}{k_{1}^{3}} \Delta^{2}\left(k_{1}, a\right) \int_{0}^{\infty} \frac{d k_{2}}{k_{2}^{3}} \Delta^{2}\left(k_{2}, a\right)\left(\frac{k_{1}^{2}}{k_{2}^{2}}+\frac{k_{2}^{2}}{k_{1}^{2}}\right) J^{(0)}\left(k_{1}, k_{2}, R\right) \\
& =\left(\frac{9}{8} a^{4} H^{4}\right)^{2} 4 \int \frac{d k_{2}}{k_{2}} \Delta^{2}\left(k_{2}, a\right) \int \frac{d k_{1}}{k_{1}^{5}} \Delta^{2}\left(k_{1}, a\right) .
\end{aligned}
$$

We will discuss the importance of this term below.

Now we turn to the variances of $\langle\varphi\rangle_{1}\left\langle\nabla^{2} \varphi\right\rangle_{1}$ and $\left\langle\nabla^{2} \varphi\right\rangle\left\langle\nabla^{2} \varphi\right\rangle$. They are given by

$$
\begin{aligned}
\operatorname{Var}\left[\langle\varphi\rangle_{1}\left\langle\nabla^{2} \varphi\right\rangle_{1}\right]= & \left(\frac{9}{4} a^{4} H^{4}\right)^{2}\left[\int \frac{d k_{1}}{k_{1}} \Delta^{2}\left(k_{1}, a\right) W^{2}\left(k_{1} R\right) \int \frac{d k_{2}}{k_{2}^{5}} \Delta^{2}\left(k_{2}, a\right) W^{2}\left(k_{2} R\right)\right. \\
& \left.+\left(\int \frac{d k}{k^{3}} \Delta^{2}(k, a) W^{2}(k R)\right)^{2}\right] \\
\operatorname{Var}\left[\left\langle\nabla^{2} \varphi\right\rangle\left\langle\nabla^{2} \varphi\right\rangle\right]= & 2\left(\frac{9}{4} a^{4} H^{4}\right)^{2}\left(\int \frac{d k}{k} \Delta^{2}(k, a) W^{2}(k R)\right)^{2} .
\end{aligned}
$$

Note that Eq. (158a also has an infrared divergence

$$
\operatorname{Var}\left[\langle\varphi\rangle_{1}\left\langle\nabla^{2} \varphi\right\rangle_{1}\right]_{\infty}=\left(\frac{9}{8} a^{4} H^{4}\right)^{2} 4 \int \frac{d k_{2}}{k_{2}} \Delta^{2}\left(k_{2}, a\right) \int \frac{d k_{1}}{k_{1}^{5}} \Delta^{2}\left(k_{1}, a\right)
$$

Of course we will be interested in the variance of the total expression Eq. (41), not the individual terms. Of particular interest is the cross term of the infrared-singular parts. This will be the only one for which we will include the cross terms. The infrared-singular pieces appear in Eq. (41) proportional to $\left\langle\varphi \nabla^{2} \varphi\right\rangle-3\langle\varphi\rangle_{1}\left\langle\nabla^{2} \varphi\right\rangle_{1}$. The infrared part of the variance of this term is

$$
\operatorname{Var}\left[\left\langle\varphi \nabla^{2} \varphi\right\rangle-3\langle\varphi\rangle_{1}\left\langle\nabla^{2} \varphi\right\rangle_{1}\right]_{\infty}=\left(\frac{9}{4} a^{4} H^{4}\right)^{2} 4 \int \frac{d k_{2}}{k_{2}} \Delta^{2}\left(k_{2}, a\right) \int \frac{d k_{1}}{k_{1}^{5}} \Delta^{2}\left(k_{1}, a\right)
$$


It is straightforward to obtain the variance for the second-order, four-derivative terms. (The sum of these terms has zero mean.) It is given by

$$
\begin{aligned}
\operatorname{Var}\left[\left\langle\left(\nabla^{2} \varphi\right)^{2}\right\rangle-\left\langle\varphi_{, i j} \varphi^{, i j}\right\rangle\right]= & \left(\frac{9}{4} a^{4} H^{4}\right)^{2} \int_{0}^{\infty} \frac{d k_{1}}{k_{1}} \Delta^{2}\left(k_{1}, a\right) \int_{0}^{\infty} \frac{d k_{2}}{k_{2}} \Delta^{2}\left(k_{2}, a\right)\left[J^{(0)}\left(k_{1}, k_{2}, R\right)-2 J^{(2)}\left(k_{1}, k_{2}, R\right)\right. \\
& \left.+J^{(4)}\left(k_{1}, k_{2}, R\right)\right]
\end{aligned}
$$

There is another potential contribution that will result in contributions to the variance similar to the terms we have found. Suppose we expand $\delta \theta$ to third order in perturbation theory. We can express $\delta \theta$ in the general form

$$
\langle\delta \theta\rangle=\left\langle\mathcal{A} \varphi+\mathcal{B} \varphi^{2}+\mathcal{C} \varphi^{3}\right\rangle
$$

where $\mathcal{A}, \mathcal{B}$, and $\mathcal{C}$ are operators which contain derivatives. Then the variance of $\delta \theta$ will contains terms like $\langle\mathcal{A} \varphi\rangle^{2}$, $\left\langle\mathcal{B} \varphi^{2}\right\rangle^{2}$, and $\langle\mathcal{A} \varphi\rangle\left\langle\mathcal{C} \varphi^{3}\right\rangle$. The first term is the usual cosmic variance term; it is well behaved in the infrared. The second term is singular in the infrared; it is given in Eq. (60). The third term will be present, in principle it also will have an infrared singular part, and its value requires a relativistic third-order perturbation calculation. However there is no reason for the infrared singular part of the $\mathcal{A C}$ term to cancel exactly the infrared singular part of the $\mathcal{B}^{2}$ term.

Finally we remark on the ultraviolet behavior of the corrections to the expansion rate. The second-order result for the mean, Eq. (50), should be well behaved in the ultraviolet. As discussed in the next section, in the linear regime $\Delta^{2}(k, a)$ increases logarithmically with $k$ for a Harrison-Zel'dovich spectrum, so the first term should be well behaved in the ultraviolet. The ultraviolet behavior of the last two terms are regulated by the filter function $W^{2}(k R)$. The contributions to the variance of the terms we have calculated, Eqs. (52b), (56), (158), and (61), all involve filter functions that regulate the ultraviolet behavior. However, we expect there to be terms that do not involve filter functions. For instance, if one performs the relativistic third-order calculation, one expects to find contributions to $\langle\delta \theta\rangle$ from terms like $\left\langle\left(\nabla^{2} \varphi\right)^{3}\right\rangle$. The variance would then include evaluation of terms like $\left\langle\nabla^{2} \varphi\right\rangle\left\langle\left(\nabla^{2} \varphi\right)^{3}\right\rangle($ an example of the aforementioned $\mathcal{A C}$ terms). These terms would include parts with a momentum integration unregulated by a filter function. If there are third-order terms with large numbers of derivatives bringing down large powers of momentum, then the variance might be sensitive to the ultraviolet behavior.

In the next section we show the numerical results for the mean and variance of the corrections to the expansion rate.

\section{NUMERICAL RESULTS}

In this section we present the numerical results for $\langle\delta \theta\rangle / 3 H$ for a matter-dominated Universe. We will give the mean values, as well as the variances.

For both the mean and the variances we express the power spectrum $\Delta^{2}(k, a)$ in terms of the transfer function $T^{2}(k)$. For a Harrison-Zel'dovich spectrum, the power spectrum is

$$
\Delta^{2}(k, a)=A^{2}\left(\frac{k}{a H}\right)^{4} T^{2}(k),
$$

where $A$ is the dimensionless amplitude, $A=1.9 \times 10^{-5}$. We will discuss the implications of other spectra. For our purposes the Bardeen, Bond, Kaiser, Szalay (BBKS) transfer function [13] will be adequate. The BBKS transfer function may be expressed in terms of a dimensionless parameter

$$
q \equiv \frac{k}{\Gamma h \mathrm{Mpc}^{-1}},
$$

where $\Gamma$ is the shape factor, defined for a flat universe in terms of the baryon fraction $\Omega_{B}$ and the total value of $\Omega_{0}$ as $\Gamma=\Omega_{0} h \exp \left(-\Omega_{B}-\sqrt{2} h \Omega_{B} / \Omega_{0}\right)$. In terms of $q$,

$$
T(q)=\frac{\ln (1+2.34 q)}{2.34 q}\left[1+3.89 q+(16.1 q)^{2}+(5.84 q)^{3}+(6.71 q)^{4}\right]^{-1 / 4}
$$

Of course at small $q, T^{2}(q) \rightarrow 1$, while at large $q, T^{2}(q) \rightarrow q^{-4} \ln ^{2} q$.

Also, in all expressions we make use of the fact that in a matter-dominated Universe $H^{2}(a)=H_{0}^{2} a_{0}^{3} / a^{3}$, where $a_{0}$ is the present value of the scale factor. 


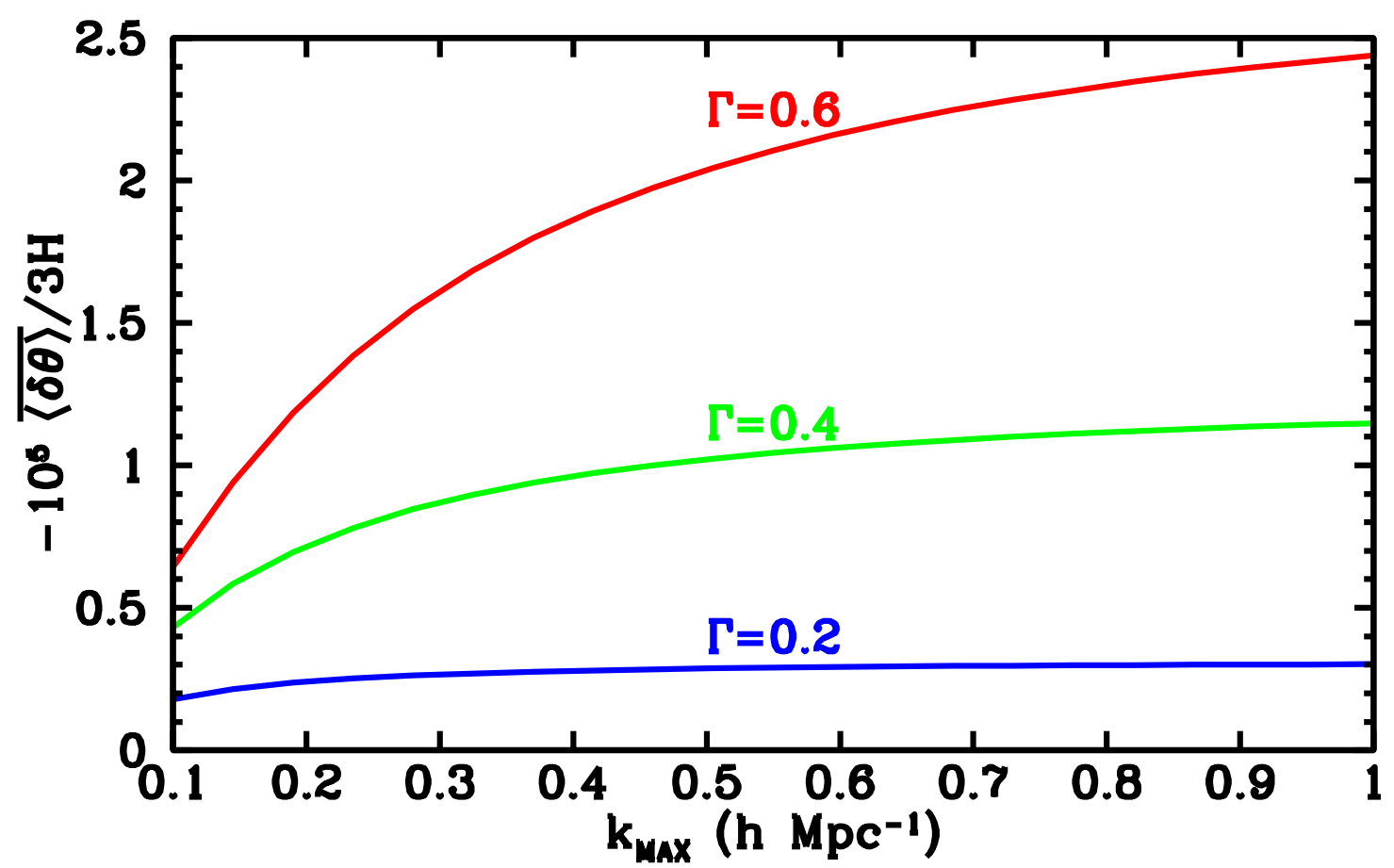

FIG. 1: The present mean value, $\overline{\langle\delta \theta\rangle} / 3 H$, in the matter-dominated Universe as a function of the ultraviolet cutoff $k_{\mathrm{MAx}}$ for various values of the shape factor $\Gamma$.

Consider the mean, given by Eq. (50):

$$
\begin{aligned}
\frac{\overline{\langle\delta \theta\rangle}}{3 H}= & -\frac{25}{24} \frac{a}{a_{0}} A^{2}\left(\frac{h \mathrm{Mpc}^{-1}}{H_{0}}\right)^{2} \Gamma^{2} \int_{0}^{\infty} d q q T^{2}(q)+\frac{25}{4} \frac{a}{a_{0}} A^{2}\left(\frac{h \mathrm{Mpc}^{-1}}{H_{0}}\right)^{2} \Gamma^{2} \int_{0}^{\infty} d q q T^{2}(q) W^{2}(r \Gamma q) \\
& -\frac{23}{96}\left(\frac{a}{a_{0}}\right)^{2} A^{2}\left(\frac{h \mathrm{Mpc}^{-1}}{H_{0}}\right)^{4} \Gamma^{4} \int_{0}^{\infty} d q q^{3} T^{2}(q) W^{2}(r \Gamma q),
\end{aligned}
$$

where $r$ is the dimensionless size of the region, $r \equiv R / h^{-1}$ Mpc. We will present results for $R=H_{0}^{-1}$ (so $r=3000$ ) and the last two terms are negligible. While we have indicated the range of integration from $q=0$ to $q=\infty$, in reality to employ Eq. (63) there is a cutoff on the maximum value of the integral. The ultraviolet cutoff arises because density perturbations become nonlinear. The mean value is very insensitive to the ultraviolet cutoff. The integral for the mean value receives most of the contribution in the decade between $10^{-1} \leq q \leq 1$.

The result for the present mean value of $\overline{\langle\delta \theta\rangle} / 3 H$ is shown in Fig. 1 for various choices of $\Gamma$. It scales as $a / a_{0}=$ $1 /(1+z)$.

Now consider the variance about the mean value. First consider the variance of the linear term, Eq. (52b). This term contributes to the variance in $\langle\delta \theta\rangle / 3 H$ an amount

$$
\left.\frac{\sqrt{\operatorname{Var}[\langle\delta \theta\rangle]}}{3 H}\right|_{\text {linear term }}=\frac{5}{9} \frac{1}{a^{2} H^{2}} \sqrt{\operatorname{Var}\left[\left\langle\nabla^{2} \varphi\right\rangle_{1}\right]}=\frac{5}{6}\left[\int_{0}^{\infty} \frac{d k}{k} \Delta^{2}(k) W^{2}(k R)\right]^{1 / 2} .
$$

Defining a dimensionless wavenumber $x=q \Gamma r$, the term becomes

$$
\left.\frac{\sqrt{\operatorname{Var}[\langle\delta \theta\rangle]}}{3 H}\right|_{\text {linear term }}=\frac{5}{6} A\left(\frac{h \mathrm{Mpc}^{-1}}{H_{0}}\right)^{2} \frac{a}{a_{0}} r^{-2}\left[\int_{0}^{x_{\mathrm{MAx}}} d x x^{3} T^{2}(x / \Gamma r) e^{-x^{2}}\right]^{1 / 2} \simeq \frac{a}{a_{0}} \frac{100}{r^{2}} \quad(\Gamma r \gg 1),
$$

where the last expression holds for $x_{\mathrm{MAX}}=k_{\mathrm{MAX}} R \gg 1$. The result is shown in Fig. 2. Because of the window function, the results do not depend on $k_{\mathrm{MAX}}$, the ultraviolet cutoff (so long as it is greater than about $k=0.1 h \mathrm{Mpc}^{-1}$ ). The 


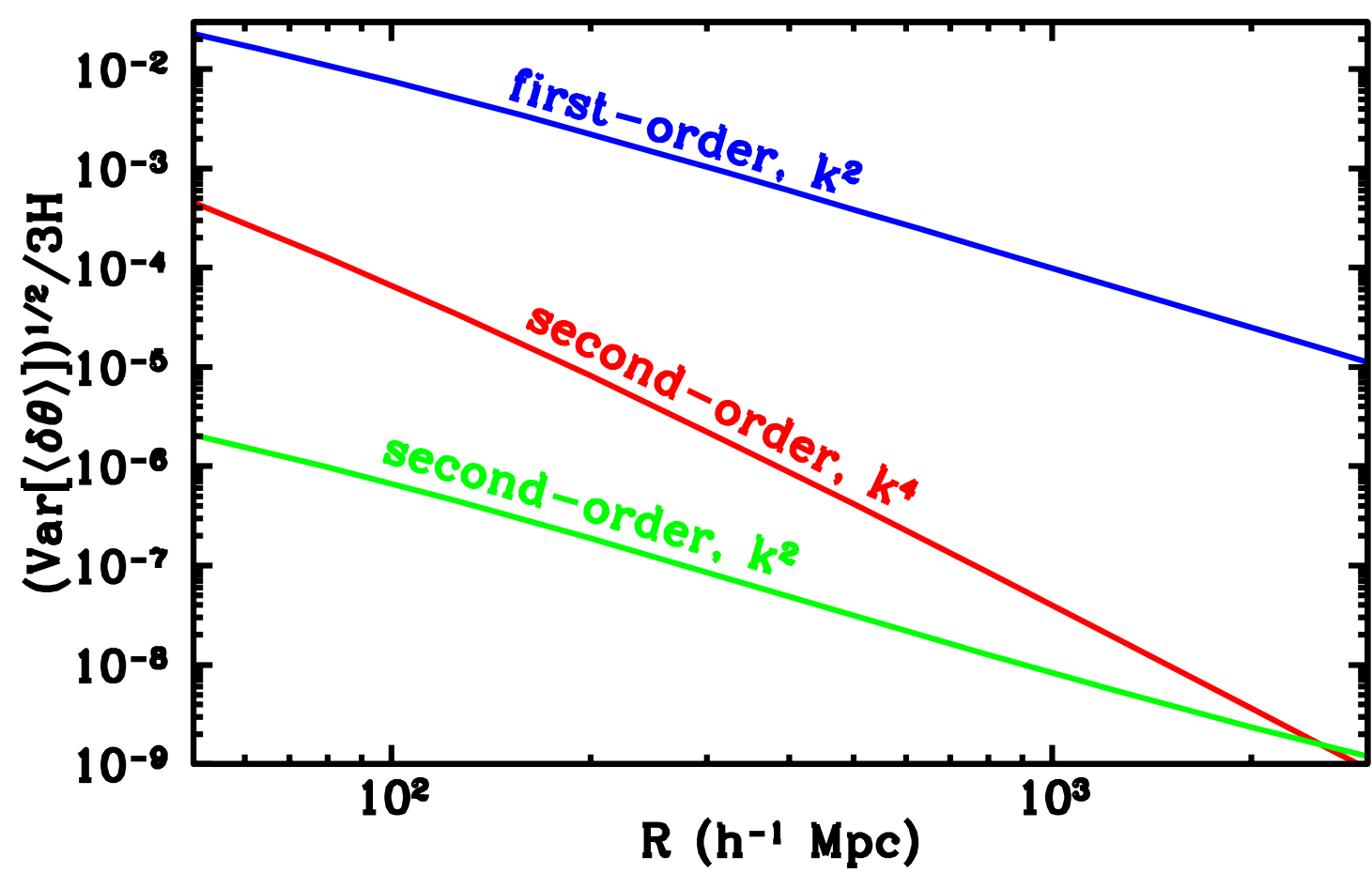

FIG. 2: The present contribution to $\sqrt{\operatorname{Var}[\langle\delta \theta\rangle]} / 3 H$ from the indicated terms in a matter-dominated Universe as a function of $R$, the volume sampled. The results are for $k_{\mathrm{MAX}}=0.1$ and $\Gamma=0.2$. On the logarithmic scale, the dependence on $\Gamma$ and $k_{\max }$ is not noticeable. We emphasize the point made in the text that the calculation for the the second-order, two-derivative term has an infrared cutoff of the present Hubble radius.

result is also well behaved in the infrared. Of course as $R \rightarrow \infty$, the variance of the linear term approaches the mean of the linear term, which is zero.

We can see comparing Fig. 1 and Fig. 2 that the variance in the linear term dominates the mean value out to distances of about $3 \times 10^{3} h^{-1} \mathrm{Mpc}$.

Next, consider the contribution to the variance of a typical second-order, four-derivative term, Eq. 61). This term contributes to the variance in $\langle\delta \theta\rangle / 3 H$ an amount

$$
\left.\frac{\sqrt{\operatorname{Var}[\langle\delta \theta\rangle]}}{3 H}\right|_{\text {second-order, } k^{4}}=\frac{4}{27} \frac{1}{a^{4} H^{4}} \sqrt{\operatorname{Var}\left[\left\langle\left(\nabla^{2} \varphi\right)^{2}\right\rangle-\left\langle\varphi_{, i j} \varphi^{, i j}\right\rangle\right]} .
$$

Again, with the same dimensionless variables

$$
\begin{aligned}
\left.\frac{\sqrt{\operatorname{Var}[\langle\delta \theta\rangle]}}{3 H}\right|_{\text {second-order, } k^{4}}= & \frac{1}{3} A^{2}\left(\frac{h \mathrm{Mpc}^{-1}}{H_{0}}\right)^{4}\left(\frac{a}{a_{0}}\right)^{2} r^{-4}\left[\int_{0}^{x_{\mathrm{MAx}}} d x_{1} \int_{0}^{x_{\mathrm{MAx}}} d x_{2} x_{1}^{3} x_{2}^{3}\right. \\
& \left.\times T^{2}\left(x_{1} / \Gamma r\right) T^{2}\left(x_{2} / \Gamma r\right) e^{-x_{1}^{2}} e^{-x_{2}^{2}} \int_{-1}^{1} d \mu e^{-2 x_{1} x_{2} \mu}\left(1-\mu^{2}\right)^{2}\right]^{1 / 2} .
\end{aligned}
$$

The results are also shown in Fig. [2] This term scales as $r^{-4}$. The result is well behaved in the infrared and an infrared cutoff does not need to be introduced.

Finally, consider the variance of a typical second-order two-derivative term, Eq. (56). As mentioned in the previous section this term is not well behaved in the infrared. We first calculate the variance by introducing an infrared cutoff which we will take to be the wavenumber of the present Hubble radius. The second-order $k^{2}$ term contributes to the 
variance in $\langle\delta \theta\rangle / 3 H$ an amount

$$
\left.\frac{\sqrt{\operatorname{Var}[\langle\delta \theta\rangle]}}{3 H}\right|_{\text {second-order, } k^{2}}=\frac{25}{27} \frac{1}{a^{2} H^{2}} \sqrt{\operatorname{Var}\left[\left\langle\varphi \nabla^{2} \varphi\right\rangle+\frac{13}{18}\left\langle\varphi, i \varphi^{, i}\right\rangle\right]} .
$$

In terms of the dimensionless variables $x$ and $r$, this term contributes to the variance an amount

$$
\begin{aligned}
\left.\frac{\sqrt{\operatorname{Var}[\langle\delta \theta\rangle]}}{3 H}\right|_{\text {second-order, } k^{2}}= & \frac{25}{24} A^{2}\left(\frac{h \mathrm{Mpc}^{-1}}{H_{0}}\right)^{2} \frac{a}{a_{0}} r^{-2}\left[\int_{x_{\mathrm{MIN}}}^{x_{\mathrm{MAX}}} d x_{1} \int_{x_{\mathrm{MIN}}}^{x_{\mathrm{MAx}}} d x_{2} T^{2}\left(x_{1} / \Gamma r\right) T^{2}\left(x_{2} / \Gamma r\right) e^{-x_{1}^{2}} e^{-x_{2}^{2}}\right. \\
& \left.\times \int_{-1}^{1} d \mu e^{-2 x_{1} x_{2} \mu}\left(\frac{x_{1}^{3}}{x_{2}}+\frac{x_{2}^{3}}{x_{1}}+2 x_{1} x_{2}+\frac{26}{9} x_{1}^{2} \mu+\frac{26}{9} x_{2}^{2} \mu+\frac{169}{81} x_{1} x_{2} \mu^{2}\right)\right]^{1 / 2}
\end{aligned}
$$

As a result of the infrared behavior, for this term we must introduce another parameter, $x_{\mathrm{MIN}}=k_{\mathrm{MIN}} R$, as an infrared cutoff. Its value is shown in Fig. 2 as a function of $R$. As expected, it has a $r^{-2}$ behavior.

Now we turn to the infrared-singular part of the second-order two-derivative term. The contribution is

$$
\left.\frac{\sqrt{\operatorname{Var}[\langle\delta \theta\rangle]}}{3 H}\right|_{\text {second-order, } k^{2}}=\frac{25}{27} \frac{1}{a^{2} H^{2}} \sqrt{\left[\left\langle\varphi \nabla^{2} \varphi\right\rangle-3\langle\varphi\rangle_{1}\left\langle\nabla^{2} \varphi\right\rangle_{1}\right]_{\infty}} .
$$

We will evaluate the integral by defining some $x_{*}=k_{*} R$ such that $x_{\text {MIN }} \ll x_{*}<1$. We will be interested in large values of $r$, so if $x \ll 1$, then $x / \Gamma r \ll 1$, and $T^{2}(x / \Gamma r)$ can be set to unity. Using Eq. (60), this term may be written as

$$
\left.\frac{\sqrt{\operatorname{Var}[\langle\delta \theta\rangle]}}{3 H}\right|_{\text {second-order, } k^{2}}=\frac{25}{12} A^{2}\left(\frac{h \mathrm{Mpc}^{-1}}{H_{0}}\right)^{2} \frac{a}{a_{0}} r^{-2}\left[x_{*}^{4} \ln \frac{k_{*}}{k_{\mathrm{MIN}}}\right]^{1 / 2} .
$$

If we take $k_{*}=k_{H}$ where $k_{H}=H=3000^{-1} h \mathrm{Mpc}^{-1}$ is the Hubble wavenumber, then evaluating the term for $r$ corresponding to the Hubble radius we find

$$
\left.\frac{\sqrt{\operatorname{Var}[\langle\delta \theta\rangle]}}{3 H}\right|_{\text {second-order, } k^{2}}=\frac{25}{24} A^{2} \frac{a}{a_{0}}\left[\ln \frac{k_{H}}{k_{\mathrm{MIN}}}\right]^{1 / 2}=\frac{25}{36} A \frac{a}{a_{0}} \sqrt{\operatorname{Var}\left[\langle\varphi\rangle_{1}\right]} .
$$

We started with a perturbative expansion, and for that expansion to be valid requires $\varphi<1$, so our perturbative analysis will break down for $\sqrt{\operatorname{Var}[\langle\delta \theta\rangle]} / 3 H \sim 10^{-5}$.

Of course the total variance of $\langle\delta \theta\rangle / 3 H$ includes cross terms from the various contributions. However we have seen that the linear term will dominate.

\section{CONCLUSIONS}

Our results are illustrated in Figs 1 and 2 The mean corrections to the expansion rate are a few parts in $10^{5}$. If the correct prescription for dealing with the infrared singular nature of $\operatorname{Var}[\varphi]$ is to employ a cutoff of the order of the Hubble radius, then the variance about the mean is small, and dominated by the first-order term. If, however, the super-Hubble modes are physical, then the variance is dominated by the infrared singular part of the second-order corrections. Restricting ourselves to $\operatorname{Var}[\varphi]<1$ where the perturbative calculation is valid, then the variance will be small, of order $10^{-5}$.

In Ref. [5], Räsänen considered what amounts to the variance associated with the second-order term proportional to $\nabla^{2} \varphi \nabla^{2} \varphi$ and suggested that due to unknown boundary conditions it may lead to a large contribution to the expansion rate. We claim that when properly averaged, this term is subdominant.

In closing, let us speculate that corrections to the expansion rate will include a term like $\varphi \nabla^{2} \varphi$ even when $\varphi \gg 1$. Returning to the infrared-singular part of the second-order variance, Eq. (75),

$$
\left.\frac{\sqrt{\operatorname{Var}[\langle\delta \theta\rangle]_{\infty}}}{3 H}\right|_{\text {second-order, } k^{2}}=3.76 \times 10^{-10} \frac{a}{a_{0}} \ln \frac{k_{H}}{k_{\mathrm{MIN}}}
$$


For the variance to be of order unity, the perturbation spectrum would have to extend to a factor of $\exp \left(6 \times 10^{18}\right)$ $\left(10^{18.8} e\right.$-folds!) times the present Hubble radius.

Rather than a Harrison-Zel'dovich spectrum, if we assume a slightly red spectrum so that $\Delta^{2}(k) \propto k^{4-\epsilon}$ with $0<\epsilon \ll 1,{ }^{7}$ then the logarithmic term in Eq. (76) is replaced by $\epsilon^{-1}\left(k_{H} / k_{\min }\right)^{\epsilon}$. Now this will give unit variance if $\ln k_{H} / k_{\mathrm{MIN}}=(43.3+\ln \epsilon) / \epsilon$. For instance, if $n=0.94$ on super-Hubble-radius scales, then a variance of order unity is obtained if the perturbation spectrum extends $676 e$-folds beyond the Hubble radius. Since the present Hubble radius corresponds to a scale that crossed the Hubble radius about $60 e$-folds before the end of inflation, if inflation lasted more than $736 e$-folds with a super-Hubble-radius spectral index of $n=0.94$, then the effect of super-Hubbleradius perturbations would have a large effect on the expansion rate of our Hubble volume. Speculation about the significance of this result will follow in a separate paper [6].

\section{Acknowledgments}

E.W.K. is supported in part by NASA grant NAG5-10842 and by the Department of Energy. We would like to thank Enrico Barausse, Riccardo Catena, Scott Dodelson, Lam Hui, Luigi Pilo, Syksy Räsänen, and Dominik Schwarz for helpful conversations.

\section{APPENDIX A: ON THE PRESCRIPTION FOR $T_{\mu \nu}^{(2)}$ AND $g_{\mu \nu}^{(2)}$}

The aim of this appendix is to demonstrate that $\langle\theta\rangle$ does not depend on the value of $\left\langle g_{\mu \nu}^{(2)}\right\rangle$ since second-order terms will only enter as spatial derivatives. For the demonstration, we first introduce $\vartheta^{i}{ }_{j}$, the extrinsic curvature of constant- $\tau$ hypersurfaces:

$$
\vartheta_{j}^{i}=\frac{1}{2} \gamma^{i k} \gamma_{k j}^{\prime}
$$

In either the synchronous or comoving gauge one can see that (see Ref. [8])

$$
\theta^{(1)}+\theta^{(11)}+\theta^{(2)}=\frac{\vartheta^{i}}{a} \equiv \frac{\vartheta}{a} .
$$

One can now write the $G^{0}$ term in a convenient form (see Eq. (4.3) of []$]$ )

$$
G_{00}^{(1)}+G_{00}^{(11)}+G_{00}^{(2)}=\frac{1}{2}\left(4 \frac{a^{\prime}}{a} \vartheta+{ }^{3} \mathcal{R}+\vartheta^{2}-\vartheta^{i}{ }_{j} \vartheta^{j}{ }_{i}\right),
$$

where ${ }^{3} \mathcal{R}$ is the intrinsic curvature of the three space with metric $\gamma_{i j}$. At second order ${ }^{3} \mathcal{R}$ is

$$
\begin{aligned}
{ }^{3} \mathcal{R}= & \gamma_{, \ell k}^{(1) \ell k}-\gamma_{k, \ell}^{(1) k, \ell}+\frac{1}{2}\left(\gamma_{, \ell k}^{(2) \ell k}-\gamma_{k, \ell}^{(2) k, \ell}\right)+\gamma^{(1) j k}\left(\nabla^{2} \gamma_{j k}^{(1)}+\gamma_{\ell, j k}^{(1) \ell}-2 \gamma_{j, \ell k}^{(1) \ell}\right) \\
& +\gamma_{, \ell}^{(1) \ell k}\left(\gamma_{j, k}^{(1) j}-\gamma_{j k}^{(1), j}\right)+\frac{3}{4} \gamma_{, k}^{(1) \ell j} \gamma_{\ell j}^{(1), k}-\frac{1}{2} \gamma_{, k}^{(1) \ell j} \gamma_{j, \ell}^{(1) k}-\frac{1}{4} \gamma_{j}^{(1) j, \ell} \gamma_{k, \ell}^{(1) k} .
\end{aligned}
$$

Using Eq. (A2), Eq. A3 also can be written as

$$
G_{00}^{(1)}+G_{00}^{(11)}+G_{00}^{(2)}=\frac{1}{2}\left[4 a^{\prime}\left(\theta^{(1)}+\theta^{(11)}+\theta^{(2)}\right)+{ }^{3} \mathcal{R}^{(1)}+{ }^{3} \mathcal{R}^{(11)}+{ }^{3} \mathcal{R}^{(2)}+\left(\vartheta^{(1)}\right)^{2}-\vartheta^{(1) i}{ }_{j} \vartheta^{(1) j}{ }_{i}\right] .
$$

Recall now that in any of the definitions adopted, Eq. (37) is always valid. So independent of $\left\langle g_{\mu \nu}^{(2)}\right\rangle$,

$$
\langle\theta\rangle=3 H-\frac{\left\langle G_{00}^{(1)}+G_{00}^{(11)}+G_{00}^{(2)}\right\rangle}{2 a^{2} H}+\left\langle\theta^{(1)}\right\rangle+\left\langle\theta^{(11)}\right\rangle+\left\langle\theta^{(2)}\right\rangle .
$$

\footnotetext{
${ }^{7}$ In the the usual parlance, this corresponds to a scalar spectral index of $1-n=\epsilon$.
} 
Using Eq. (A5), we obtain

$$
\langle\theta\rangle=3 H-\frac{1}{4 a^{2} H}\left[\left\langle{ }^{3} \mathcal{R}^{(1)}+{ }^{3} \mathcal{R}^{(11)}\right\rangle+\left\langle{ }^{3} \mathcal{R}^{(2)}\right\rangle+\left\langle\left(\vartheta^{(1)}\right)^{2}-\vartheta^{(1) i}{ }_{j} \vartheta^{(1) j}{ }_{i}\right\rangle\right] .
$$

We note now that the only piece that depends on $g_{\mu \nu}^{(2)}$ is the one contained in ${ }^{3} \mathcal{R}^{(2)}$. And, as we can see from its explicit expression

$$
{ }^{3} \mathcal{R}^{(2)}=\frac{1}{2}\left(\gamma_{, l k}^{(2) l k}-\gamma_{, l l}^{(2) k k}\right)
$$

this is a spatial gradient, so independent on $\left\langle g_{\mu \nu}^{(2)}\right\rangle$. From this formula it is also clear that $\theta$ has no term with zero powers of $k$, since the spatial curvature ${ }^{3} \mathcal{R}$ always involves spatial derivatives (and the same is true for the $\left\langle\left(\vartheta^{(1)}\right)^{2}\right\rangle-\left\langle\vartheta_{j}^{(1) i} \vartheta_{i}^{(1) j}\right\rangle$ terms $)$.

If one computes $\langle\theta\rangle$ using Eq. (A7), one obtains the same result as Eq. (41), thus our results are independent of $\left\langle g_{\mu \nu}^{(2)}\right\rangle$. By the same argument, in a first-order calculation we could add a constant to $g_{\mu \nu}^{(1)}$.

\section{APPENDIX B: $\theta$ IN THE POISSON GAUGE}

In this appendix we repeat the calculation in the Poisson gauge in order to check our results and compare with other results in the literature for $\theta$ in the that gauge. ${ }^{8}$ The Poisson gauge is a second-order generalization of the longitudinal (or Newtonian) gauge. Since this is a very involved way to do the computation, and since it is only intended to be a check, we do it only in a partial way by just computing the corrections $\theta^{(1)}+\theta^{(11)}+\theta^{(2)}$ [see Eqs. (18) and (39)] . Furthermore we compute exactly the terms with two spatial derivatives, while as for the four derivatives terms, we only check that they have zero statistical mean (that is, we disregard total spatial gradients).

The metric is given at second order in this gauge by ${ }^{9}$

$$
d s^{2}=a_{P}^{2}(\eta)\left\{-\left(1+2 \phi^{(1)}+\phi^{(2)}\right) d \eta^{2}+\omega_{i}^{(2)} d y^{i} d \eta+\left[\left(1-2 \psi^{(1)}-\psi^{(2)}\right) \delta_{i j}+\frac{1}{2} \chi_{i j}^{(2)}\right] d y^{i} d y^{j}\right\} .
$$

Here $\chi_{i j}^{(2)}$ is a pure symmetric, transverse, traceless tensor degree of freedom. We will denote the derivative with respect to conformal time $\eta$ as $\partial_{\eta}$. We will also introduce here a cosmic time $z$, linked to $\eta$ through the relation $d z=a d \eta$, and we will denote the derivative with respect to $z$ with a $\partial_{z}$ symbol. The spatial derivatives with respect to $x^{i}$ will be denoted as before, with a simple $\partial^{i}$ or ${ }^{i}$ symbol. The derivatives with respect to $y^{i}$ instead will be explicitly written as $\partial / \partial y^{i}$ or with the subscript $\partial_{(\mathbf{y})}^{i}$. Recall also, as well known, that the $i-i$ component of Einstein's equations (in the absence of anisotropic stress) imposes $\phi^{(1)}=\psi^{(1)}$, and moreover, the evolution equations in matter domination give

$$
\phi^{(1)}\left(z, y^{i}\right)=\psi^{(1)}\left(z, y^{i}\right)=\varphi\left(y^{i}\right) .
$$

The four velocity of the fluid $u^{\mu}$ here has a more involved form

$$
\begin{aligned}
u^{0} & =\frac{1}{a_{P}}\left(1-\varphi+\frac{3}{2} \varphi^{2}+\frac{2}{9 \mathcal{H}_{P}^{2}} \partial_{(\mathbf{y}) i} \varphi \partial_{(\mathbf{y})}^{i} \varphi-\frac{\phi^{(2)}}{2}\right), \\
u^{i} & =\frac{1}{a_{P}}\left(-\frac{2}{3 \mathcal{H}_{P}} \partial_{(\mathbf{y})}^{i} \varphi+\frac{v^{(2) i}}{2}\right),
\end{aligned}
$$

where $\mathcal{H}_{P} \equiv a_{P}^{-1} \partial_{\eta} a_{P}$. Here $v^{(2) i}$ is the second-order contribution to the three velocity. We will need only its divergence (which is obtained taking the divergence of the $0-i$ Einstein equation):

$$
\begin{aligned}
\partial_{(\mathbf{y}) i} v^{(2) i} & =\frac{4}{3 \mathcal{H}_{P}}\left(-\partial_{(\mathbf{y})}^{i} \varphi \partial_{(\mathbf{y})_{i}} \varphi-\varphi \nabla_{(\mathbf{y})}^{2} \varphi+\frac{2\left(\partial_{(\mathbf{y})}^{i} \varphi \nabla_{(\mathbf{y})}^{2} \partial_{(\mathbf{y}) i} \varphi\right)}{3 \mathcal{H}_{P}^{2}}+\frac{2\left(\nabla_{(\mathbf{y})}^{2} \varphi\right)^{2}}{3 \mathcal{H}_{P}^{2}}\right) \\
& -\frac{2}{3 \mathcal{H}_{P}}\left(\nabla_{(\mathbf{y})}^{2} \phi^{(2)}+\frac{\nabla_{(\mathbf{y})}^{2} \psi^{(2) \prime}}{\mathcal{H}}\right) .
\end{aligned}
$$

\footnotetext{
8 Actually, the other results we are aware of in the literature use only the first-order metric.

${ }^{9}$ Also here, for our purposes the vector $\omega_{i}^{(2)}$ and the tensor $\chi_{i j}^{(2)}$ will never enter in the computations.
} 
Now, taking these expressions and taking Christoffel symbols from Ref. 11], we must compute

$$
\theta=\partial_{\mu} u^{\mu}+\Gamma_{\mu \alpha}^{\alpha} u^{\mu}=\partial_{\eta} u^{0}+\partial_{(\mathbf{y}) i} u^{i}+\Gamma_{\mu \alpha}^{\alpha} u^{\mu} .
$$

This gives us

$$
\theta\left(z, y^{i}\right)=3 H_{P}-3 H_{P} \varphi+\frac{9 H \varphi^{2}}{2}+\frac{20 \partial_{(\mathbf{y}) i} \varphi \partial_{(\mathbf{y})}^{i} \varphi}{9 a_{P}^{2} H_{P}}-\frac{3 H \phi^{(2)}}{2}-\frac{3 \dot{\psi}^{(2)}}{2}+\partial_{(\mathbf{y}) i} u^{(1) i}+\partial_{(\mathbf{y}) i} u^{(2) i} .
$$

Here $H_{P}$ is defined as $H_{P} \equiv a_{P}^{-1} \partial_{z} a_{P}=a_{P}^{-2} \partial_{\eta} a_{P}=a_{P}^{-1} \mathcal{H}_{P}$. The piece coming from $\partial_{(\mathbf{y}) i} u^{i}$ in Eq. (B5) contains first order $\left(\partial_{(\mathbf{y}) i} u^{(1) i}\right)$ and second order $\left(\partial_{(\mathbf{y}) i} u^{(2) i}\right)$ terms. The first order term [see Eq. (B3b)] is equal to

$$
\partial_{(\mathbf{y}) i} u^{(1) i}=-\frac{2}{3 a_{P}^{2} H_{P}} \nabla_{(\mathbf{y})}^{2} \varphi
$$

and it will be important in the next steps of the computation, since it will produce second-order quantities. The term $\partial_{(\mathbf{y}) i} u^{(2) i}[\mathrm{Eq}$. (B4)] is a spatial gradient of second-order quantities. For the purposes of this appendix we have to keep the first two pieces in Eq. (B4), and moreover we have to consider also the two intrinsically second-order terms $\phi^{(2)}$ and $\psi^{(2)}$. Note also that, in their time evolution they contain non-local quantities $\left(\Theta_{0}\right.$ and $\Psi_{0}$ of Eqs. $(6.8)$ of Ref. [8]), i.e., they are defined through

$$
\begin{aligned}
& \nabla_{(\mathbf{y})}^{2} \Theta_{0}=\Psi_{0}-\frac{1}{3} \partial_{(\mathbf{y}) i} \varphi \partial_{(\mathbf{y})}^{i} \varphi \\
& \nabla_{(\mathbf{y})}^{2} \Psi_{0}=-\frac{1}{2}\left[\left(\nabla_{(\mathbf{y})}^{2} \varphi\right)^{2}-\partial_{(\mathbf{y}) j} \partial_{(\mathbf{y}) k} \varphi \partial_{(\mathbf{y})}^{j} \partial_{(\mathbf{y})}^{k} \varphi\right] .
\end{aligned}
$$

So the spatial derivatives of these quantities will produce not only spatial gradients, but also terms who do not have zero statistical mean. In the end, we keep them in the calculation as

$$
\partial_{i} u^{(2) i}=-\frac{\nabla_{(\mathbf{y})}^{2} \phi^{(2)}}{3 a_{P}^{2} H_{P}}-\frac{\nabla_{(\mathbf{y})}^{2} \dot{\psi}^{(2)}}{3 a_{P}^{2} H_{P}^{2}}-\frac{2}{3 a_{P}^{2} H_{P}}\left(\partial_{(\mathbf{y})}^{i} \varphi \partial_{(\mathbf{y})_{i}} \varphi+\varphi \nabla_{(\mathbf{y})}^{2} \varphi\right)+\cdots
$$

where "..." indicates terms with four spatial gradients (that we will omit).

So, the relevant expression for $\theta\left(z, y^{i}\right)$ is obtained by Eqs. (B6), (B7), and (B9)

$$
\begin{aligned}
\theta\left(z, y^{i}\right) & =3 H_{P}-3 H_{P} \varphi+\frac{9 H \varphi^{2}}{2}+\frac{20 \partial_{(\mathbf{y}) i} \varphi \partial_{(\mathbf{y})}^{i} \varphi}{9 a_{P}^{2} H_{P}}-\frac{2}{3 a_{P}^{2} H_{P}}\left(\partial_{(\mathbf{y})}^{i} \varphi \partial_{(\mathbf{y})_{i}} \varphi+\varphi \nabla_{(\mathbf{y})}^{2} \varphi\right) \\
& -\frac{3 H \phi^{(2)}}{2}-\frac{3 \dot{\psi}^{(2)}}{2}-\frac{2 \nabla_{(\mathbf{y})}^{2} \varphi}{3 a_{P}^{2} H_{P}}-\frac{\nabla_{(\mathbf{y})}^{2} \phi^{(2)}}{3 a_{P}^{2} H_{P}}-\frac{\nabla_{(\mathbf{y})}^{2} \dot{\psi}^{(2)}}{3 a_{P}^{2} H_{P}^{2}}+\cdots
\end{aligned}
$$

This is the result in the Poisson gauge in coordinates $\left(z, y^{i}\right)$. Note that here there are terms which do not vanish in the infrared limit, proportional to $\varphi$ and in $\varphi^{2}$ (they will disappear going back to the synchronous and comoving coordinates, as noted in Ref. [12]).

In order to compare with the results already obtained in the synchronous gauge, we have to express our quantity $\theta\left(z, y^{i}\right)$ as $\theta\left(t, x^{i}\right)$ and average it over a volume in coordinates $x^{i}$ at constant $\tau$ as in Section III First, we have to change time from $z$ to $t$ in all the quantities. This is relevant only for the zeroth-order and first-order terms, and not for the ones which are already second order. So the quantities in which the time has to be changed are $H_{P}, a_{P}(z)$ and $\varphi\left(z, y^{i}\right)$. The first two, $a_{P}(z)=\left(z / z_{0}\right)^{2 / 3}$ and $H_{P}=2 / 3 z$, have to be expanded up to second order in $\delta t$, which is defined by $t=z+\delta t$ and expressed in terms of the scale factor and the Hubble rate defined by a comoving observer: $a=\left(t / z_{0}\right)^{2 / 3}, H=2 / 3 t$. Instead, for the variable $\varphi\left(\eta, y^{i}\right), \varphi\left(\eta, y^{i}\right)=\varphi\left(\tau, x^{i}\right)$ holds since $\partial_{\eta} \varphi\left(\eta, y^{i}\right)=0$. In order to find the change of the time coordinate from $z$ to $t$ one knows that $u^{\mu} \partial_{\mu}=\partial_{t}$, and that $\partial_{t} t=1$. In this way one obtains an iterative equation

$$
\left(1+v^{(1) 0}+v^{(2) 0}\right) \frac{d}{d z}\left(z+\delta t^{(1)}+\delta t^{(2)}\right)+u^{(1) i} \partial_{(\mathbf{y}) i}\left(z+\delta t^{(1)}\right)=1,
$$

where $u^{\mu} \equiv a_{P}^{-1}\left(\delta_{0}^{\mu}+v^{(1)}+v^{(2)}\right)$. For zeroth, first, and second order, the equation becomes

$$
\begin{aligned}
1 & =\frac{d z}{d z} \\
0 & =v^{(1) 0}+\frac{d}{d z} \delta t^{(1)} \\
0 & =v^{(2) 0}+v^{(1) 0} \frac{d}{d z} \delta t^{(1)}+\frac{d}{d z} \delta t^{(2)}+u^{(1) i} \partial_{(\mathbf{y}) i} \delta t^{(1)}
\end{aligned}
$$


which lead to

$$
\begin{aligned}
\delta t^{(1)} & =\varphi t \\
\delta t^{(2)} & =-\frac{1}{2} \varphi^{2} t+\frac{4 \varphi_{, i} \varphi^{, i}}{45 a^{2} H}+\frac{1}{2} \int^{t} d t^{\prime} \phi^{(2)} .
\end{aligned}
$$

Now using these equations, one can change the time in Eq. (B10), obtaining

$$
\theta\left(t, y^{i}\right)=3 H+\frac{118 \varphi^{(\mathbf{y}), i} \varphi_{(\mathbf{y}), i}}{45 a^{2} H}-\frac{3 H \phi^{(2)}}{2}-\frac{3 \dot{\psi}^{(2)}}{2}+\frac{9 H^{2} \int^{\tau} \phi^{(2)}}{4}+\partial_{(\mathbf{y}) i} u^{i} .
$$

As said before, this transformation (i.e., passing to synchronous time $t$ ) has eliminated the two terms in $\varphi$ and $\varphi^{2}$ that do not vanish in the infrared. We wrote it in this form in order to compare with a previous result in literature (Eq. (10) of Ref. 5]). As we are going to show, we agree with that result, but we added here the contribution of intrinsic ${ }^{10}$ second-order terms $\phi^{(2)}$ and $\psi^{(2)}$. This is crucial if we want to recover the result obtained in Sec. III in the other gauge. Here $\partial_{(\mathbf{y}) i} u^{i}$ contains the terms

$$
\partial_{(\mathbf{y}) i} u^{i}=-\frac{2 \nabla_{(\mathbf{y})}^{2} \varphi}{3 a^{2} H}-\frac{2 \varphi \nabla_{(\mathbf{y})}^{2} \varphi}{9 a^{2} H}-\frac{2}{3 a^{2} H}\left(\partial_{(\mathbf{y})}^{i} \varphi \partial_{(\mathbf{y})_{i}} \varphi+\varphi \nabla_{(\mathbf{y})}^{2} \varphi\right)-\frac{\nabla_{(\mathbf{y})}^{2} \phi^{(2)}}{3 a^{2} H}-\frac{\nabla_{(\mathbf{y})}^{2} \dot{\psi}^{(2)}}{3 a^{2} H^{2}}+\cdots
$$

Note that after changing time, a new term (which is not a spatial gradient) appears, namely $-2 \varphi \nabla_{(\mathbf{y})}^{2} \varphi / 9 a^{2} H$.

Finally, one has to change also spatial variables from $y^{i}$ to $x^{i}$. The change of coordinates is found by imposing that the metric $g_{\mu \nu}$ in the coordinates $\left(t, x^{i}\right)$ has $g_{0 i}=0$. Using the transformation given by Eq. (B13), one finds that to first order the change in spatial coordinates is given by

$$
x^{i}=y^{i}+\frac{2}{3} \frac{\partial_{(\mathbf{y})}^{i} \varphi}{a^{2} H} .
$$

Since it is already first order, this change of coordinates is irrelevant for quantities that are already second-order. Moreover, the only first order term of Eq. (B14) is $\partial_{(\mathbf{y}) i} u^{(1) i}=-2 \nabla_{(\mathbf{y})}^{2} \varphi / 3 a^{2} H$. Here, the change of spatial coordinates in the argument of $\varphi\left(\tau, y^{i}\right)$ is not relevant for our purposes, since it produces only an extra term which is a four derivative spatial gradient of something. But passing from $\nabla_{(\mathbf{y})}^{2}$ to $\nabla^{2}$ produces a (relevant) extra term with four spatial derivatives,

$$
-\frac{2}{3 a^{2} H} \nabla_{(\mathbf{y})}^{2} \varphi=-\frac{2}{3 a^{2} H} \nabla^{2} \varphi-\frac{4 \varphi^{, j k} \varphi, j k}{9 a^{4} H^{3}} .
$$

In all the other terms we can safely put $\partial_{(\mathbf{y})}=\partial$. After doing this, the result is

$$
\begin{aligned}
\theta\left(t, x^{i}\right)= & 3 H-\frac{2 \nabla^{2} \varphi}{3 a^{2} H}-\frac{8 \varphi \nabla^{2} \varphi}{9 a^{2} H}+\frac{88 \varphi^{, i} \varphi, i}{45 a^{2} H}-\frac{4 \varphi^{, j k} \varphi, j k}{9 a^{4} H^{3}}- \\
& \frac{3 H \phi^{(2)}}{2}-\frac{3 \dot{\psi}^{(2)}}{2}+\frac{9 H^{2} \int^{t} \phi^{(2)}}{4}-\frac{\nabla^{2} \phi^{(2)}}{3 a^{2} H}-\frac{\nabla^{2} \dot{\psi}^{(2)}}{3 a^{2} H^{2}}+\cdots
\end{aligned}
$$

Using Eqs. (6.8) of Ref. [8], together with Eqs. (B8) given in the present section, one obtains for the time evolution of the intrinsic second order terms $\phi^{(2)}$ and $\psi^{(2)}$ the result

$$
\theta\left(t, x^{i}\right)=3 H-\frac{2 \nabla^{2} \varphi}{3 a^{2} H}+\frac{5 \varphi^{, i} \varphi_{, i}}{3 a^{2} H}-\frac{40 \varphi \nabla^{2} \varphi}{9 a^{2} H}-\frac{4 \varphi^{, j k} \varphi, j k}{9 a^{4} H^{3}}+\cdots
$$

Now this is the first check we wanted to do. We can compare with Eq. (39) and see that deriving by parts and using $a H=2 / \tau$, they coincide (up to four-derivative total spatial gradients).

An important observation here is that the presence of the intrinsically second-order terms is necessary to obtain not only the correct result, but even a consistent calculation. In fact if one ignores them, i.e., ignores $\psi^{(2)}$ and $\chi^{(2)}$ in the synchronous gauge and ignores $\phi^{(2)}$ and $\psi^{(2)}$ in the Poisson gauge, and compares the two results, one sees that they

10 Note however that what is "intrinsic" second order in a gauge, can become first order times first order in another one. 
do not coincide. In other words they are not really "intrinsic" second order, since what appears as intrinsic second order in one gauge, can appear as first order times first order in another gauge.

At this point we may also average expression Eq. (B19) over a volume $d^{3} x$ as we did in Sec. III This has to be done with the appropriate measure of integration, which is $d^{3} x \sqrt{\gamma}$, where $\gamma \equiv \operatorname{det}\left(g_{i j}\right)$. Up to first order the spatial metric is given by

$$
g_{i j}=\frac{\partial y^{\alpha}}{\partial x^{i}} \frac{\partial y^{\beta}}{\partial x^{j}} \tilde{g}_{\alpha \beta}=a_{P}^{2}\left[\delta_{i j}(1-2 \varphi)-\frac{4}{3 a^{2} H^{2}} \partial_{i} \partial_{j} \varphi\right]=a^{2}\left(1-\frac{4}{3} \varphi\right)\left[\delta_{i j}(1-2 \varphi)-\frac{4}{3 a^{2} H^{2}} \partial_{i} \partial_{j} \varphi\right],
$$

where $\tilde{g}$ is the metric in the $z, y$ coordinates, and where we used the transformation of Eq. (B16). Using this metric, the measure becomes

$$
d^{3} y \sqrt{\gamma}=d^{3} y a^{3}\left[1-5 \varphi-\frac{2}{3 a^{2} H^{2}} \nabla^{2} \varphi\right]
$$

and this is the same as Eq. (6), and so the final result is the same.

Finally, we can check the computation of Ref. [5], which used only the first order metric in this gauge. That is, we have to put $\phi^{(2)}$ and $\psi^{(2)}$ to zero in Eq. (BB18),

$$
\begin{aligned}
\theta\left(t, x^{i}\right) & =3 H-\frac{2 \nabla^{2} \varphi}{3 a^{2} H}-\frac{8 \varphi \nabla^{2} \varphi}{9 a^{2} H}+\frac{88 \varphi^{, i} \varphi_{, i}}{45 a^{2} H}-\frac{4 \varphi^{, j k} \varphi_{, j k}}{9 a^{4} H^{3}}+\cdots \\
& =3 H-\frac{2 \nabla^{2} \varphi}{3 a^{2} H}+\frac{128 \varphi^{, i} \varphi, i}{45 a^{2} H}-\frac{8 \partial_{i}\left(\varphi \partial^{i} \varphi\right)}{9 a^{2} H}-\frac{4 \varphi^{, j k} \varphi, j k}{9 a^{4} H^{3}}+\cdots,
\end{aligned}
$$

and average it with the measure of Eq. B21). Doing so, we recover the result

$$
\langle\theta\rangle(t)=3 H-\frac{22}{45} \frac{\left\langle\partial^{i} \varphi \partial_{i} \varphi\right\rangle}{a^{2} H}+\frac{22}{9} \frac{\left\langle\partial_{i}\left(\varphi \partial^{i} \varphi\right)\right\rangle}{a^{2} H}+\cdots,
$$

as in Eq. (11) of Ref. [5].

[1] W. L. Freedman, et al., Ap. J. 553, 47 (2001).

[2] A. G. Riess et al., Astron. J. 116, 1009 (1998); S. Perlmutter et al., Ap. J. 517, 565 (1999).

[3] L. Hui and U. Seljak, in Proceedings of the 173rd IAU Symposium, edited by C. S. Kochanek and J. N. Hewitt (Kluwer Academic Publishers, Dordrecht, 1995); U. Seljak and L. Hui, in Proceedings of Clusters, Lensing and the Future of the Universe (ASP Conference Series vol. 88, V. Trimble and A. Reisenegger, eds., 1995), p 267.

[4] G. Bene, V. Czinner, and M. Vasuth, astro-ph/0308161

[5] S. Räsänen, JCAP 0402, 003 (2004).

[6] E. W. Kolb, S. Matarrese, A. Notari, and A. Riotto, in preparation.

[7] A. R. Liddle and D. H. Lyth, Phys. Rep. 231, 1 (1993).

[8] S. Matarrese, S. Mollerach and M. Bruni, Phys. Rev. D 58, 043504 (1998).

[9] T. Buchert, C. Sicka, Phys. Rev. D 62, 043525 (2000).

[10] N. Bartolo, S. Matarrese and A. Riotto, JHEP 0404, 006 (2004).

[11] V. Acquaviva, N. Bartolo, S. Matarrese and A. Riotto, Nucl. Phys. B 667, 119 (2003).

[12] G. Geshnizjani and R. Brandenberger, Phys. Rev. D 66, 123507 (2002).

[13] J. M. Bardeen, J. R. Bond, N. Kaiser, and A. S. Szalay, Ap. J. 321, 28 (1986). 